\title{
The evolving jet spectrum of the neutron star X-ray binary AqI X-1 in transitional states during its 2016 outburst
}

\author{
M. Díaz Trigo ${ }^{1}$, D. Altamirano ${ }^{2}$, T. Dinçer ${ }^{3}$, J. C. A. Miller-Jones ${ }^{4}$, D. M. Russell ${ }^{5}$, A. Sanna ${ }^{6}$, C. Bailyn ${ }^{3}$, \\ F. Lewis ${ }^{7,8}$, S. Migliari ${ }^{9,10}$, and F. Rahoui ${ }^{11}$ \\ 1 ESO, Karl-Schwarzschild-Strasse 2, 85748 Garching bei München, Germany \\ e-mail: mdiaztri@eso.org \\ 2 Department of Physics \& Astronomy, University of Southampton, Southampton, Hampshire SO17 1BJ, UK \\ 3 Department of Astronomy, Yale University, PO Box 208101, New Haven CT 06520-8101, USA \\ ${ }^{4}$ International Centre for Radio Astronomy Research, Curtin University, GPO Box U1987 Perth, Western Australia 6845, Australia \\ 5 New York University Abu Dhabi, PO Box 129188, Abu Dhabi, UAE \\ 6 Dipartimento di Fisica, Università degli Studi di Cagliari, SP Monserrato-Sestu km 0.7, 09042 Monserrato, Italy \\ 7 Faulkes Telescope Project, School of Physics and Astronomy, Cardiff University, 5 The Parade, Cardiff CF24 3AA, UK \\ 8 Astrophysics Research Institute, Liverpool John Moores University, 146 Brownlow Hill, Liverpool L3 5RF, UK \\ 9 XMM-Newton Science Operations Centre, ESAC/ESA, Camino Bajo del Castillo s/n, Urb. Villafranca del Castillo, \\ 28691 Villanueva de la Cañada, Madrid, Spain \\ 10 Institute of Cosmos Sciences, University of Barcelona, Martí i Franquès 1, 08028 Barcelona, Spain \\ 11 Department of Astronomy, Harvard University, 60 Garden street, Cambridge MA 02138, USA
}

Received 24 January 2018 / Accepted 20 April 2018

\section{ABSTRACT}

\begin{abstract}
We report on quasi-simultaneous observations from radio to X-ray frequencies of the neutron star X-ray binary Aql X-1 over accretion state transitions during its 2016 outburst. All the observations show radio to millimetre spectra consistent with emission from a jet, with a spectral break from optically thick to optically thin synchrotron emission that decreases from $\sim 100 \mathrm{GHz}$ to $<5.5 \mathrm{GHz}$ during the transition from a hard to a soft accretion state. The $5.5 \mathrm{GHz}$ radio flux density as the source reaches the soft state, $0.82 \pm 0.03 \mathrm{mJy}$, is the highest recorded to date for this source. During the decay of the outburst, the jet spectral break is detected again at a frequency of $\sim 30-100 \mathrm{GHz}$. The flux density is $0.75 \pm 0.03 \mathrm{mJy}$ at $97.5 \mathrm{GHz}$ at this stage. This is the first time that a change in the frequency of the jet break of a neutron star X-ray binary has been measured, indicating that the processes at play in black holes are also present in neutron stars, supporting the idea that the internal properties of the jet rely most critically on the conditions of the accretion disc and corona around the compact object, rather than the black hole mass or spin or the neutron star surface or magnetic field.
\end{abstract}

Key words. X-rays: binaries - ISM: jets and outflows - accretion, accretion disks - stars: neutron - X-rays: individuals: Aq1 X-1

\section{Introduction}

Relativistic jets from supermassive black holes (BHs) at the centres of galaxies and stellar-mass BHs in X-ray binaries (XRBs) have been extensively studied for decades. However, key questions including where and why they are formed, how they are collimated and whether the energy comes from the accretion disc or the spin of the $\mathrm{BH}$ remain unanswered. Observationally, it has been established that the presence/absence of a compact or transient jet in $\mathrm{BH}$ XRBs is related to the accretion flow geometry during a transient outburst (Fender et al. 2004). A steady jet is present from the beginning of the outburst, when the source is in the so-called "hard" state. The jet persists while the luminosity rises and until the X-ray spectrum starts to soften. During the transition to the "soft" state, the steady jet emission is quenched (see e.g. Russell et al. 2011; Coriat et al. 2011) and in some sources discrete ejecta are launched and their radio emission can be spatially resolved (Mirabel \& Rodriguez 1994; Tingay et al. 1995); although these ejections are not found in every transition (Paragi et al. 2013). When the BH transitions back to the hard state at a lower luminosity, the compact jet is reinstated (Kalemci et al. 2005, 2013).

In the last years, major efforts have been devoted to understanding what determines the frequency of the jet spectral break from optically thick to optically thin emission (Gandhi et al. 2011; van der Horst et al. 2013; Corbel et al. 2013; Russell et al. 2013, 2014; Koljonen et al. 2015). This break together with the cooling break expected at higher energies (Sari et al. 1998) are proxies for the total radiative power of the jet and may provide important information for understanding jet launching and quenching. Russell et al. (2013, 2014) performed multiwavelength observations of the BH XRB MAXI J1836-194 during its 2011 outburst. They found that the spectral break from optically thick to optically thin emission moved from infrared (IR) to radio frequencies as the spectrum got softer at a fairly constant luminosity during a spectral state transition and then moved back up again as the spectrum got harder at the end of the outburst. This indicates that the main player in determining the break frequency is the changing structure of the accretion flow, rather than the mass and spin of the $\mathrm{BH}$ or its luminosity.

The temporal evolution of the optically thick-to-thin jet break has never been studied over the course of a neutron star (NS) XRB outburst. In fact, studies of NS jets have so far been relatively sparse, due to their faintness in the radio band (see Migliari \& Fender 2006 and references therein). However, such studies are of fundamental importance since they offer the possibility to isolate the role played by the $\mathrm{BH}$ event horizon or the NS surface or magnetic field in powering the jets. 
Aql X-1 is a NS (Koyama et al. 1981) low-mass X-ray binary (LMXB) undergoing accretion outbursts every 200-300 days. Its distance was determined from type I X-ray bursts to be 4.5-6 kpc (Jonker \& Nelemans 2004) ${ }^{1}$. Based on its spectral and timing properties, it was classified as an atoll-type X-ray binary (Reig et al. 2004). Casella et al. (2008) observed millisecond X-ray pulsations with a frequency of $550.27 \mathrm{~Hz}$ that were identified with the NS spin frequency. The pulsations only lasted for $150 \mathrm{~s}$ and were never detected again, highlighting the possibility of transient pulsations in any of the "non-pulsating" XRBs. The optical counterpart of Aql X-1 was identified as a K7V star by Chevalier et al. (1999). Recently, Mata Sánchez et al. (2017) used the Very Large Telescope (VLT)/SINFONI to obtain time resolved spectra for both Aql X-1 and the interloper star located less than $0.5^{\prime \prime}$ from Aql X-1, and classified the optical counterpart to Aql X-1 as a $\mathrm{K} 4 \pm 2$ star moving at a projected velocity of $136 \pm 4 \mathrm{~km} \mathrm{~s}^{-1}$. They determined its orbital inclination to be $36-47^{\circ}$, lower than that of $72-79^{\circ}$ determined by Galloway et al. (2016) based on the detection of two dipping episodes in the RXTE X-ray light curves. The orbital period of the binary was determined to be $19 \mathrm{~h}$ (Chevalier \& Ilovaisky 1991; Welsh et al. 2000) from the light curve modulations in quiescence.

Radio emission from AqlX-1 was first detected with the Very Large Array (VLA) during its 1990 outburst with flux densities of 0.4 and $0.13 \mathrm{mJy}$ (Hjellming et al. 1990). Tudose et al. (2009) analysed the March 2002 outburst and did not find any significant correlation between the radio and optical $(R$-band) emission, consistent with Maitra \& Bailyn (2008) who attributed the optical-infrared (OIR) emission to thermal heating of the irradiated outer accretion disc. Miller-Jones et al. (2010) investigated the radio-X-ray coupling during the entire 2009 outburst and found that in the hard state it is qualitatively similar to that found for BHs, in that the radio and X-ray fluxes positively correlate. Moreover, they found that during the soft state, the radio emission was quenched to $\lesssim 0.2 \mathrm{mJy}$ for luminosities $\gtrsim 10 \%$ Eddington.

Maitra \& Bailyn (2008) first classified the outbursts of Aql X-1 in two types depending on the OIR light curve morphology: the fast rise and exponential decay (FRED) type outburst seen in many soft X-ray transients, and the low-intensity state (LIS), where the optical-to-soft X-ray flux ratio is much higher than during a FRED. They did not find a single correlation between the optical ( $R$-band) and soft X-ray fluxes suggesting that those two types of outbursts have fundamentally different accretion flow properties. The OIR light curves did not show any signature of compact jets or accretion state changes. According to this classification, between June 1998 and November 2007 only three outbursts were included in the FRED-only category, namely those that occurred on October 2000, March 2002, and March 2003.

Asai et al. (2012) also classified the Aq1 X-1 outbursts but based on the relative intensity evolutions below/above $15 \mathrm{keV}$. They identified slow (S-type) and fast (F-type) outbursts, the former having a longer initial hard state $(\gtrsim 9 d)$ than the latter $(\lesssim 5 \mathrm{~d})$. Importantly, for the S-type, the $15-50 \mathrm{keV}$ intensity reaches the maximum before the hard-to-soft transition, while the opposite is true for the F-type (see their Fig. 8). They also found that the S-type outbursts had higher luminosity both before the start of the outburst and at the hard-to-soft state transition, and concluded that the X-ray irradiation during the pre-outburst period would determine whether the outburst becomes S-type or F-type.

\footnotetext{
1 Hereafter, we adopt a distance of $5.2 \mathrm{kpc}$ when calculating luminosities.
}

In the S-type, the hard-to-soft state transition occurred at $\sim 5 \%$ of the Eddington luminosity. Examples of S-type outbursts in this classification occurred on November 2009, September 2010 and October 2011.

Finally, Güngör et al. (2014) made a slightly different classification based on the duration and maximum flux of the X-ray emission and identified three types of outbursts: long-high (including the October 2000 FRED outburst and the 2011 S-type outburst), medium-low (February 2002 and September 2010) and short-low (November 2009). They also concluded that irradiation is the dominant physical process that leads to the different classes of outbursts.

Following the exploratory Atacama Large Millimeter/ submillimeter Array (ALMA) study of NSs by Díaz Trigo et al. (2017), we successfully proposed for quasi-simultaneous observations of a NS transient at radio, millimetre ( $\mathrm{mm}$ ), IR and X-ray wavelengths. The goal was to study the behaviour of the jet break over the transitional states for the first time in a NS LMXB and to test the hypothesis that the jet break moves to lower frequencies as the source evolves towards softer states, as was observed for the BH LMXB MAXIJ1836-194 (Russell et al. 2013, 2014). In NSs, jet spectral breaks lying in the IR band have been reported in three sources (Migliari et al. 2010; Baglio et al. 2016; Díaz Trigo et al. 2017) but we expect large changes in the frequency of the jet break during state transitions, based on the behaviour of MAXI J1836-194. We triggered our multiwavelength campaign in August and September 2016, during a recent outburst of Aql X-1. In this paper, we report on the results of this campaign.

\section{Observations and data analysis}

We observed AqlX-1 during August and September 2016 at radio, $\mathrm{mm}$, IR, optical and $\mathrm{X}$-ray wavelengths with the Australia Telescope Compact Array (ATCA), ALMA, the VLT, the SMARTS $1.3 \mathrm{~m}$ telescope, the Faulkes telescopes, Las Cumbres Observatory (LCO) telescopes and Swift (Gehrels et al. 2004), respectively. We also used one contemporaneous NUSTAR observation for analysis. Table 1 shows a log of the radio, $\mathrm{mm}$, mid-IR and Swift X-ray pointed observations.

Figure 1 shows the light curve of the 2016 outburst as covered by Swift and the Faulkes/LCO optical telescopes including the times of the radio, $\mathrm{mm}$, and mid-IR pointed observations presented here. To generate the Swift/XRT light curves we estimated the average-per-observation intensity (count rate in the $0.3-10 \mathrm{keV}$ band) and hardness ratio (HR, ratio of the count rates in the $1.5-10 \mathrm{keV}$ and $0.3-1.5 \mathrm{keV}$ energy bands) using the online light curve generator provided by the UK Swift Science Data Centre (UKSSDC; Evans et al. 2007). The Swift/BAT data are provided by the Swift Team as daily averages in the energy range 15-50 keV (Krimm et al. 2013).

\subsection{ATCA}

We made four ATCA observations of Aql X-1, on 2, 4 and 7 August and 19 September, under program code C3010 (see Table 1). All observations were taken simultaneously in two 2-GHz frequency bands, centred at 5.5 and 9.0 GHz. The three observations in August were taken in the very compact H75 array configuration, and the September observation was made in the slightly more extended H168 configuration. These configurations are both hybrid arrays, with maximum baselines for the inner five antennas of 89 and $192 \mathrm{~m}$, respectively, and the sixth antenna located $4.4 \mathrm{~km}$ away and of little use for direct imaging. 
Table 1. Observation log.

\begin{tabular}{lcc}
\hline \hline Observatory & Start time (UT) & End time (UT) \\
\hline Obs 1 & & \\
ATCA 5.5 \& 9 GHz & 02 Aug 2016 10:34 & 02 Aug 2016 15:30 \\
ALMA 97.49 GHz & 03 Aug 2016 01:39 & 03 Aug 2016 02:06 \\
ALMA 302.99 GHz & 03 Aug 2016 00:35 & 03 Aug 2016 01:38 \\
VLT/VISIR & 03 Aug 2016 00:27 & 03 Aug 2016 01:26 \\
Swift/XRT & 03 Aug 2016 03:03 & 03 Aug 2016 03:30 \\
Obs 2a & & \\
ATCA 5.5 \& 9 GHz & 04 Aug 2016 09:27 & 04 Aug 2016 14:59 \\
ALMA 302.99 GHz & 05 Aug 2016 05:47 & 05 Aug 2016 06:51 \\
VLT/VISIR & 04 Aug 2016 02:20 & 04 Aug 2016 04:15 \\
Swift/XRT & 05 Aug 2016 01:53 & 05 Aug 2016 03:27 \\
Obs 2b & & \\
ATCA 5.5 \& 9 GHz & 07 Aug 2016 12:16 & 07 Aug 2016 16:30 \\
ALMA 97.49 GHz & 07 Aug 2016 03:49 & 07 Aug 2016 04:19 \\
Swift/XRT & 07 Aug 2016 17:18 & 07 Aug 2016 17:34 \\
NuSTAR & 07 Aug 2016 11:04 & 08 Aug 2016 05:18 \\
Obs 3 & & \\
ATCA 5.5 \& 9 GHz & 19 Sep 2016 06:00 & 19 Sep 2016 11:00 \\
ALMA 97.49 GHz & 19 Sep 2016 22:10 & 19 Sep 2016 22:36 \\
ALMA 302.99 GHz & 20 Sep 2016 00:32 & 20 Sep 2016 01:35 \\
Swift/XRT & 19 Sep 2016 14:10 & 19 Sep 2016 14:29 \\
\hline
\end{tabular}

Notes. The single observations within each subset are ordered by increasing frequency.

These compact configurations therefore provide relatively poor angular resolution, but have the advantage of some north-south resolution due to the location of two antennas on the north spur. This makes imaging an equatorial source such as Aql X-1 much more reliable than would be the case for an east-west interferometer such as the ATCA in a more extended configuration. However, one of the inner antennas was missing in each of the four observations, reducing the quality and sensitivity of our imaging. We used 1934-638 as an amplitude and bandpass calibrator, and the nearby compact source $1849+005$ to calibrate the antenna complex gains. Data reduction was carried out using standard procedures within the Multichannel Image Reconstruction, Image Analysis and Display (MIRIAD; Sault et al. 1995) software package, and then imported into the Common Astronomy Software Application package (CASA; McMullin et al. 2008) for imaging.

The August observations had angular resolutions of $\sim 120^{\prime \prime} \times$ $60^{\prime \prime}$ at $5.5 \mathrm{GHz}$, and $\sim 75^{\prime \prime} \times 40^{\prime \prime}$ at $9.0 \mathrm{GHz}$, improving to $\sim 55^{\prime \prime} \times 27^{\prime \prime}$ and $\sim 33^{\prime \prime} \times 18^{\prime \prime}$, respectively, in September. Due to the poor angular resolution and the sparse $u v$-coverage we undertook a number of steps to extract the most accurate flux densities for Aql X-1. Firstly, we subtracted a confusing source 43.7" west of Aql X-1, at (J2000) 19:11:13.40+00:34:47.7, with a flux density of $240 \mu \mathrm{Jy}$ at $5.25 \mathrm{GHz}$ and a spectral index of $\alpha=-1.08$, which was detected in higher-resolution archival VLA observations (A. Deller, private communication) but due to the compact array configuration was not resolved in our images, except for the September observation at $9 \mathrm{GHz}$. For this observation, we measured the flux density of the confusing source to be $134 \pm 15 \mu \mathrm{Jy}$, fully consistent with the $134 \mu \mathrm{Jy}$ expected at $9 \mathrm{GHz}$ from the measured VLA spectrum. Therefore, we confirm that the confusing source is not significantly variable and that the procedure used is adequate to fully remove it from our data. Secondly, we created an image of the field stacking all four epochs and subtracted all sources out to a distance of $17^{\prime}$ from the phase centre in the $u v$-plane after refining their positions using the NRAO VLA Sky Survey (NVSS) catalogue where available (Condon et al. 1998). Finally, we extracted flux densities using two methods: the conventional image-plane analysis, and directly fitting the target in the $u v$-plane using the UVMULTIFIT algorithm of Martí-Vidal et al. (2014), expected to be more reliable when the $u v$-coverage is poor. After checking that the values were broadly consistent for both methods, we adopted the values obtained with the second method (the $u v$ plane fitting) and list them in Table 2.

Aql X-1 was detected at all four ATCA epochs, at both 5.5 and $9.0 \mathrm{GHz}$. The emission initially dropped between 2 and 4 August, before peaking on 7 August at $810 \pm 19 \mu \mathrm{Jy}$ at $5.5 \mathrm{GHz}$ and $528 \pm 19 \mu \mathrm{Jy}$ at $9.0 \mathrm{GHz}$.

\subsection{ALMA}

ALMA observed Aq1 X-1 four times from August to September 2016 (see Table 1). All observations were set up to use four spectral windows, each with a bandwidth of $2 \mathrm{GHz}$. The spectral windows were centred at 90.49, 92.43, 102.49 and $104.49 \mathrm{GHz}$ for the three observations in band 3 and at 295.99, 297.93, 307.99 and $309.99 \mathrm{GHz}$ for the observations in band 7. The spectral resolution for each spectral window was $31.2 \mathrm{MHz}$. The observations were performed using between 38 and $4612-\mathrm{m}$ antennas. The maximum baseline length was 1.4 and $3.1 \mathrm{~km}$, for the August and September observations, respectively. The bandpass calibrator was J1751+0939 for all band 3 observations and the first band 7 observation and J1924-2914 for the second and third band 7 observations. The bandpass calibrator was also used as flux calibrator for all observations except the second band 3 observation, for which J1733-1304 was used. The phase calibrator was $\mathrm{J} 1851+0035$ for all observations except the last band $3 \mathrm{ob}-$ servation, for which $\mathrm{J} 1907+0127$ was used.

We re-generated images from the ALMA calibrated products following standard procedures with CASA v4.7. For the band 7 observations, the images extracted with the initial calibrated products showed slightly extended emission. This could be due to loss of phase coherence since the weather conditions were marginal for observations at frequencies $\gtrsim 300 \mathrm{GHz}$. Therefore, we performed a detailed check of the calibrated products and found significant phase variability within a calibrator scan for antennas further than 260, 400 and $400 \mathrm{~m}$ from the centre of the array for obs $1,2 \mathrm{a}$ and 3 , respectively. We re-calibrated the datasets for these observations flagging out the problematic antennas before re-generating the images. These images show point-like source emission, similarly to the band 3 observations. Notably, the measured flux densities increased by $\gtrsim 50 \%$ relative to the original pipeline-calibrated images, highlighting the improvement in the quality of the images. Consequently, we consider the images and flux densities obtained after re-calibration hereafter.

Figure 2 shows the final reconstructed images at the position of Aql X-1, extracted with Briggs weighting and robust $=0.5$ for all the observations and Table 2 lists the flux densities for all observations.

At $97.49 \mathrm{GHz}$ (band 3), the synthesized beam size was between $0.46^{\prime \prime} \times 0.35^{\prime \prime}$ and $0.71^{\prime \prime} \times 0.50^{\prime \prime}$, depending on the epoch. The peak of radio emission is centred on average at (J2000) $19: 11: 16.024 \pm 0.005+00: 35: 05.900 \pm 0.007$ and the peak flux densities are $636 \pm 24,406 \pm 26$ and $753 \pm 29 \mu \mathrm{Jy}$ for epochs 1 , $2 \mathrm{~b}$ and 3 , respectively. The reported flux density for all sources in this section corresponds to the peak flux from the elliptical 


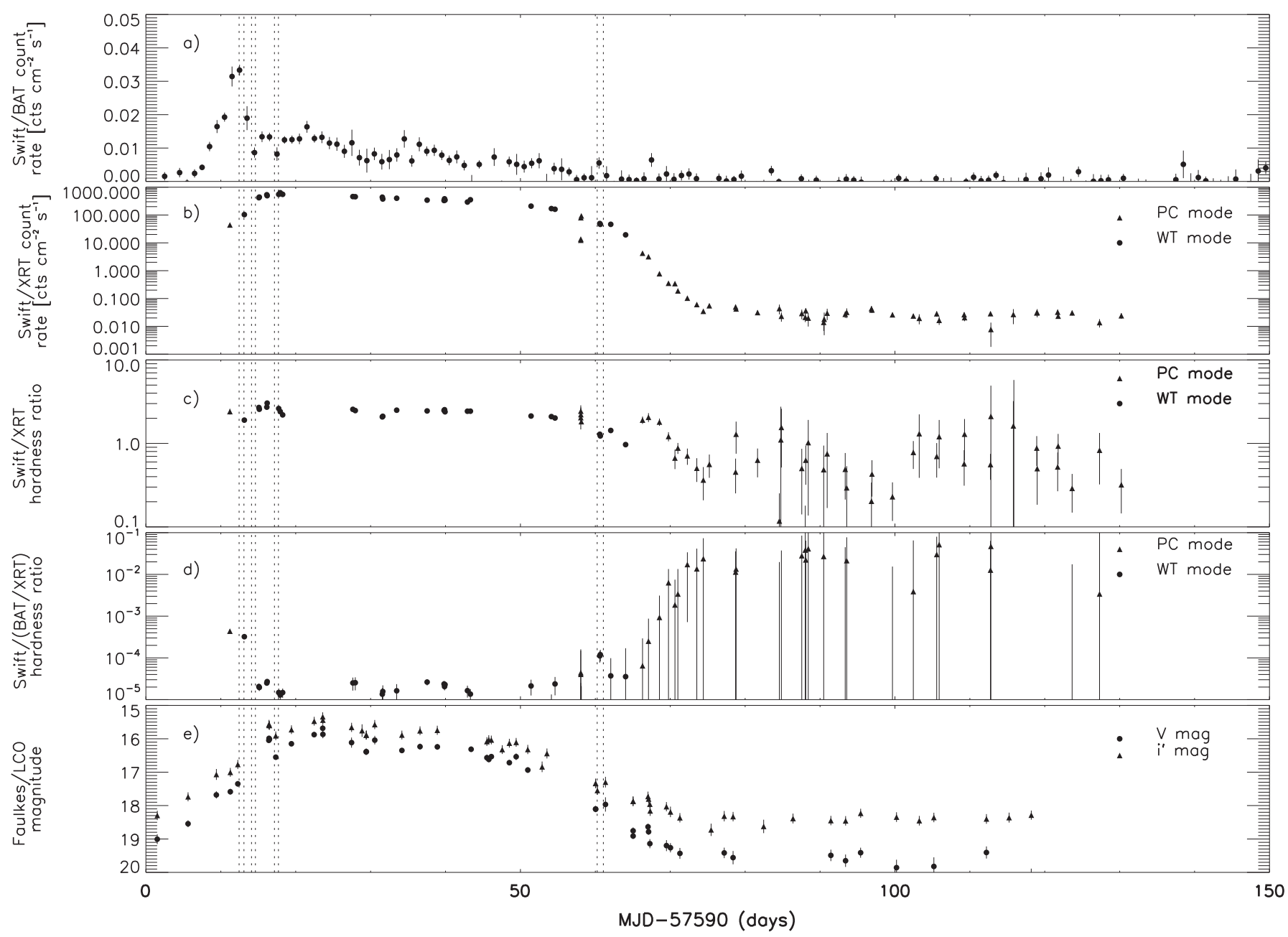

Fig. 1. X-ray and optical light curves of AqlX-1. The start and end times of the four multi-wavelength observations listed in Table 1 are indicated with dotted lines. The start of each multi-wavelength observation is defined as the start time of the first observation within a set; for example, the start of the ATCA/VLT observations mark the start of obs 1/2a, respectively. Similarly, the end of each multi-wavelength observation is defined as the end time of the last observation within a set; for example, the end of the Swift/ALMA observations mark the end of obs 1/2a, respectively. Panel a: Swift/BAT 15-50 keV count rate. Panel b: Swift/XRT $0.3-10 \mathrm{keV}$ count rate (count rates in PC mode could be affected by pile-up at count rates above $\sim 1 \mathrm{cts} \mathrm{s}^{-1}$ ). Panel $c$ : Swift/XRT hardness ratio $(1.5-10 \mathrm{keV} / 0.3-1.5 \mathrm{keV}$ count rate). Panel $d$ : Swift/(BAT/XRT) hardness ratio $(15-50 \mathrm{keV} / 0.3-10 \mathrm{keV}$ count rate). The hardness ratio is calculated for days on which there is a Swift/XRT point and a Swift/BAT point within one day. Panel $e$ : Faulkes/LCO V and $i^{\prime}$ magnitudes.

Gaussian fitted to the source emission in the image plane and the error corresponds to the rms of the image measured far away from the source position.

At $302.99 \mathrm{GHz}$ (band 7), the synthesized beam size was between $0.37^{\prime \prime} \times 0.33^{\prime \prime}$ and $0.58^{\prime \prime} \times 0.58^{\prime \prime}$, depending on the epoch. The peak of radio emission is centred on average at (J2000) 19:11:16.02 $\pm 0.01+00: 35: 05.89 \pm 0.01$ and the peak flux density is $532 \pm 42$ and $598 \pm 63 \mu \mathrm{Jy}$ for obs 1 and 3 , respectively. During obs $2 \mathrm{a}$, we do not detect the source and the $3 \sigma$ upper limit is $<189 \mu \mathrm{Jy}$.

\subsection{VLT/SMARTS/LCO}

Mid-IR observations of the field of AqlX-1 were made with the VLT on 3 and 4 August 2016 under program 097.D-0803 (PI: Russell). The VLT Imager and Spectrometer for the mid-IR (VISIR; Lagage et al. 2004) instrument on UT3 (Melipal) was used in small-field imaging mode, with a pixel scale of 45 mas pixel $^{-1}$. These and more observations near the end of September will be fully presented in Russell et al. (in prep.), which focusses on the evolution of the mid-IR to UV spectral energy distributions (SEDs) in detail. The August observations were performed in the B10.7 (centred at $10.65 \mu \mathrm{m}$ ) filter on 3 August and the J8.9 $(8.72 \mu \mathrm{m})$ and B11.7 $(11.52 \mu \mathrm{m})$ filters on 4 August. Half of the approximately one-hour observing time in each filter was on source, due to the chop-nod mode. The data were reduced using the VISIR pipeline. Raw images from the chop/nod cycle were recombined and sensitivities were estimated based on standard star observations taken on the same night.

We obtained optical and near-IR photometric observations with the dual channel imager ANDICAM (DePoy et al. 2003) on the SMARTS $1.3 \mathrm{~m}$ telescope (Subasavage et al. 2010). An observing sequence consisted of a $300 \mathrm{~s}$ exposure in $R$-band, a $600 \mathrm{~s}$ exposure in each of $V$ - and $I$-band, a $50 \mathrm{~s}$ exposure in each of five dithered images of $J$-band, and a $70 \mathrm{~s}$ exposure in each of nine dithered images of $H$-band. The data were reduced with IRAF scripts (Tody 1986, 1993) ${ }^{2}$, following the standard reduction procedures in Buxton et al. (2012). The magnitudes were put to the standard scale with respect to four nearby stars in the field, with absolute calibration via optical primary standards

2 http://iraf.net/ 
Table 2. Fluxes, flux densities and/or count rates of the observations reported.

\begin{tabular}{|c|c|c|c|c|c|c|c|c|c|}
\hline \multirow[t]{3}{*}{ Date } & \multirow{3}{*}{$\begin{array}{l}\text { MJD- } \\
57590\end{array}$} & \multicolumn{2}{|c|}{ ATCA } & \multicolumn{2}{|c|}{ ALMA } & \multicolumn{2}{|c|}{ VLT/VISIR } & \multirow{3}{*}{$\begin{array}{c}\text { Swift/XRT } \\
0.4-9 \mathrm{keV} \\
{\left[10^{-9} \mathrm{erg} \mathrm{cm}^{-2} \mathrm{~s}^{-1}\right]}\end{array}$} & \multirow{3}{*}{$\begin{array}{c}\text { Swift/BAT } \\
15-50 \mathrm{keV} \\
{\left[\mathrm{cts} \mathrm{cm}-2 \mathrm{~s}^{-1} \text { ] }\right.}\end{array}$} \\
\hline & & \multirow{2}{*}{$\begin{array}{c}5.5 \mathrm{GHz} \\
{[\mu \mathrm{Jy}]}\end{array}$} & \multirow{2}{*}{$\begin{array}{c}9 \mathrm{GHz} \\
{[\mu \mathrm{Jy}]}\end{array}$} & \multirow{2}{*}{$\begin{array}{c}97.49 \mathrm{GHz} \\
{[\mu \mathrm{Jy}]}\end{array}$} & \multirow{2}{*}{$\begin{array}{c}302.99 \mathrm{GHz} \\
{[\mu \mathrm{Jy}]}\end{array}$} & \multicolumn{2}{|c|}{$8.7-11.5 \mu \mathrm{m}$} & & \\
\hline & & & & & & {$[\mathrm{mJy}]$} & {$[\mathrm{mJy}]$} & & \\
\hline 02 Aug 2016 & $5 \quad 12$ & $515 \pm 26$ & $428 \pm 28$ & - & - & - & - & - & $0.033 \pm 0.002(d)$ \\
\hline 03 Aug 2016 & $6 \quad 13$ & - & - & $636 \pm 24$ & $532 \pm 42$ & $<1.603(\mathrm{~B} 10.7)$ & - & $5.60 \pm 0.04$ & $0.025 \pm 0.004$ \\
\hline 04 Aug 2016 & $5 \quad 14$ & $494 \pm 19$ & $208 \pm 18$ & - & - & $<1.931(\mathrm{~B} 11.7)$ & $<1.820(\mathrm{~J} 8.9)$ & - & $0.009 \pm 0.002(d)$ \\
\hline 05 Aug 2016 & $5 \quad 15$ & - & - & - & $<189$ & - & - & $23.1 \pm 0.1$ & $0.014 \pm 0.004$ \\
\hline 07 Aug 2016 & $6 \quad 17$ & $810 \pm 19$ & $528 \pm 19$ & $406 \pm 26$ & - & - & - & $28.6 \pm 0.3$ & $0.011 \pm 0.004$ \\
\hline 19 Sep 2016 & 60 & $259 \pm 13$ & $366 \pm 12$ & $753 \pm 29$ & - & - & - & $1.94 \pm 0.02$ & $0.009 \pm 0.003$ \\
\hline \multirow[t]{3}{*}{20 Sep 2016} & 61 & - & - & - & $598 \pm 63$ & - & - & - & $0.002 \pm 0.003(d)$ \\
\hline & & SMARTS $H$ & SMARTS $J$ & LCO $Y$ & SMARTS I & $\mathrm{LCO} i^{\prime}$ & SMARTS/LCO & SMARTS/LCO $V$ & $\mathrm{LCO} B$ \\
\hline & & $1.650 \mu \mathrm{m}$ & $1.250 \mu \mathrm{m}$ & $1.004 \mu \mathrm{m}$ & $0.798 \mu \mathrm{m}$ & $\begin{array}{c}0.755 \mu \mathrm{m} \\
{[\mathrm{mJy}]}\end{array}$ & $0.641 \mu \mathrm{m}$ & $0.545 \mu \mathrm{m}$ & $0.436 \mu \mathrm{m}$ \\
\hline 02 Aug 2016 & $6 \quad 12$ & - & - & - & - & 1.82 & 1.79 & 1.71 & 1.63 \\
\hline 03 Aug 2016 & $5 \quad 13$ & - & 1.69 & - & - & - & 1.82 & 1.86 & - \\
\hline 04 Aug 2016 & $5 \quad 14$ & - & 2.40 & - & 3.41 & - & 4.02 & 4.66 & - \\
\hline 05 Aug 2016 & $6 \quad 15$ & - & - & - & - & - & - & 4.66 & - \\
\hline 07 Aug 2016 & $5 \quad 17$ & 2.07 & 3.08 & 3.86 & 3.47 & 4.01 & 3.53 & 3.97 & 3.02 \\
\hline 19 Sep 2016 & 60 & 0.41 & 0.68 & 0.65 & 0.82 & 0.89 & 0.78 & 0.86 & 0.61 \\
\hline
\end{tabular}

Notes. For Swift/BAT we list either the orbital count rate closest in time to the Swift/XRT observation within a given day, or the daily count rate (d) if no Swift/XRT observations were available on the same day. For SMARTS/LCO, the errors are typically smaller than 0.1 magnitudes, making the flux errors typically smaller than $10 \%$ and smaller than the symbols in Fig. 5. Upper limits are given at a $3 \sigma$ significance level.
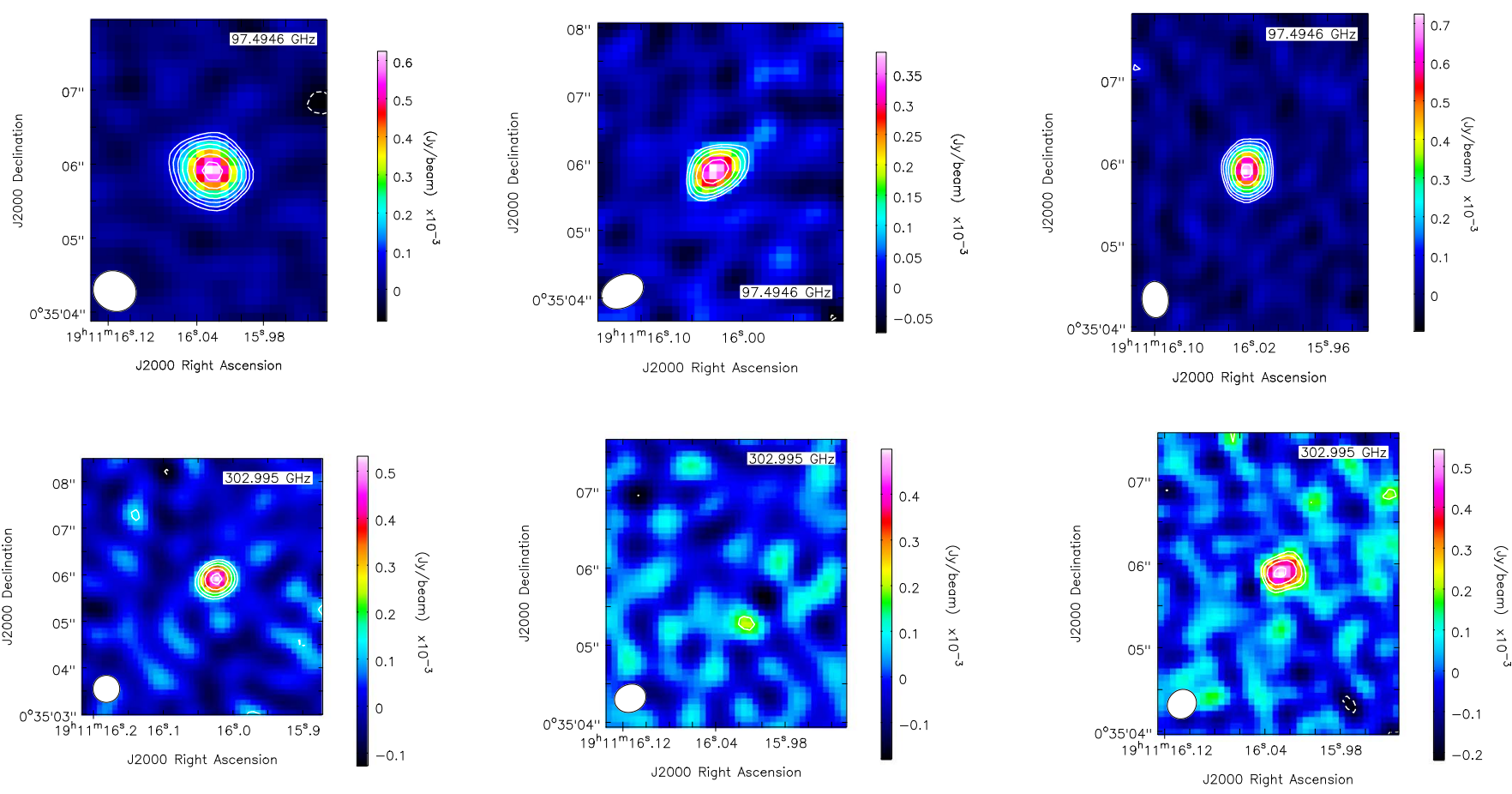

Fig. 2. ALMA images of the averaged data of Aql X-1 at $97.49 \mathrm{GHz}$ (upper panels) and $302.99 \mathrm{GHz}$ (lower panels) during epochs 1-3 (from left to right). Contours are overlaid in the zoomed image at levels of $\pm(\sqrt{2})^{n} \sigma$ significance with $n=3,4,5 \ldots 15$ with respect to the rms of each observation (see Table 2). The synthesized beam is shown at the lower-left corner of each image.

(Landolt 1992) on clear nights and the Two Microns All-Sky Survey catalog (2MASS; Skrutskie et al. 2006).

We are observing Aq1X-1 regularly with the $2 \mathrm{~m}$ robotic Faulkes Telescope North (located at Haleakala on Maui) and Faulkes Telescope South (at Siding Spring, Australia; e.g. Russell \& Lewis 2009; Russell et al. 2010), as part of an ongoing monitoring campaign of 40 LMXBs (Lewis et al. 2008). Once the 2016 outburst was first detected, we increased the cadence of observations and the number of optical filters, and also made observations with the $1 \mathrm{~m}$ LCO robotic telescope network. These included $1 \mathrm{~m}$ telescopes located at Cerro Tololo InterAmerican Observatory in Chile, McDonald Observatory in 
Texas, USA, South African Astronomical Observatory (SAAO) at Sutherland in South Africa, and Siding Spring Observatory in Australia. During the outburst, imaging data were taken in the Bessell $B, V, R$, Sloan Digital Sky Survey (SDSS) $i^{\prime}$ and PanSTARRS $Y$-band filters (see Table 2 for central wavelengths).

All Faulkes/LCO data were reduced using the LCO automatic pipeline. Photometry was performed using PHOT in IRAF. For photometric calibration we made use of field stars close to AqlX-1 with known magnitudes (with magnitude errors of $\leq 0.05$ ) tabulated in the Pan-STARRS 1 and APASS catalogues (Magnier et al. 2013; Henden et al. 2015; Chevalier et al. 1999 and Maitra \& Bailyn 2008). The resulting $V$ and $i^{\prime}$-band light curves are presented in Fig. 1e. The shape of the outburst can clearly be seen and appears to correlate well with the Swift/XRT flux in Fig. 1b. The optical colour is bluer when brighter (the $V$-band flux increases by a larger amplitude than the $i^{\prime}$-band flux), which is consistent with previous findings (e.g. Maitra \& Bailyn 2008). The LCO data in the remaining filters are used for the SEDs in Fig. 5 only.

To calculate the intrinsic OIR fluxes, we de-reddened the OIR magnitudes adopting an extinction of $A_{\mathrm{V}}=1.53 \pm 0.07 \mathrm{mag}$ and the extinction law of Cardelli et al. (1989). This extinction value is derived from the neutral hydrogen absorption column of $N_{\mathrm{H}}^{\mathrm{abs}}=(4.4 \pm 0.1) \times 10^{21} \mathrm{~cm}^{-2}$ measured by Campana et al. (2014) using a combined fit to XMM-Newton and Chandra spectra (see also Table A.1 and King et al. 2016 for similar values) and adopting the relation between $A_{\mathrm{V}}$ and $N_{\mathrm{H}}^{\text {abs }}$ given in Foight et al. (2016).

\subsection{Swift and NuSTAR}

Swift/XRT observed Aql X-1 at numerous times during its 2016 outburst, four of them coincident with the days of our multiwavelength campaign (see Table 1). We re-generated spectra from Swift/XRT observations in window timing (WT) mode following standard procedures and using the latest calibration files (March 2017; CALDB version 20170306). A type-I burst episode was recorded during observation 00033665074 and removed before spectral extraction. We next estimated the source count rate within a 30-pixel radius region (corresponding to $\sim 71^{\prime \prime}$ ), and background counts within an annular region with inner and outer radii of 33 and 50 pixels, respectively. The first three observations (00033665074, 00033665075, 00033665076 taken on 3, 5 and 7 August, respectively) showed source count rates above the nominal pile-up threshold $\left(100 \mathrm{cts} \mathrm{s}^{-1}\right.$, see e.g. Romano et al. 2006). Thus, we next extracted source spectra from annular regions with increasing inner radii and an outer radius fixed to 30 pixels and fitted them with an absorbed power-law model. We observed significant spectral distortion as a function of the inner radius of the annular region used for spectral extraction, confirming the presence of pile-up in the three examined observations. For each observation we then excised the core region until no variation of the power-law photon index was detected. The final regions used for spectral extraction have inner radii of 2 pixels $\left(4.72^{\prime \prime}\right.$, obs. 00033665074) and 6 pixels (14.16", obs. 00033665075 and 00033665076). Finally, we rebinned the source spectra to ensure a minimum of 20 counts per bin and used the $0.4-9 \mathrm{keV}$ energy range for analysis, performed using XSPEC (Arnaud 1996), version 12.9.1.

NUSTAR observed Aql X-1 on 7 August 2016 (Obs. ID. 90201024002) for a total exposure of $10 \mathrm{ks}$. We performed standard screening and filtering of the events using the NUSTAR data analysis software (nustardas) version 1.5.1.
We extracted source and background events from a circular region of radius $50^{\prime \prime}$ centred on the source position and far from the source, respectively. We then extracted source and background spectra and generated response files for all instruments (FPMA and FPMB) using the nuproducts pipeline. Finally, we rebinned the source spectra to ensure a minimum of 20 counts per bin and used the $3-30 \mathrm{keV}$ energy range for analysis.

We first fitted all the Swift/XRT observations individually. We tried the same models for all the observations despite their potentially different states for the sake of completeness. We started with one-component models like blackbody, disc blackbody, power law and thermal Comptonisation (respectively represented by the XSPEC models bbodyrad, diskbb (Mitsuda et al. 1984), po and nthcomp (Zdziarski et al. 1996; Życki et al. 1999). To account for absorption from neutral material we used the tbabs XSPEC model with ISM abundances from Wilms et al. (2000) and photo-electric cross sections from Verner et al. (1996). In Table A.1 we show the results of the fits only for those observations/models that resulted in $\chi_{v}^{2}<2$ (Models 1-3, corresponding to diskbb, po and nthcomp, respectively). Due to the limited statistics and energy range of the spectra, the $\chi_{v}^{2}$ values are already very low for the onecomponent models shown, indicating that two-component models will be subject to a large degeneracy. However, we can attempt to constrain the two-component models by fitting all the spectra simultaneously and linking the column density of the absorbing material, expected to be constant. Following previous authors (Lin et al. 2007; Sakurai et al. 2012, 2014; Ono et al. 2017), we tested four two-component models: blackbody plus power law (Model 4), disc blackbody plus power law (Model 5), blackbody plus disc blackbody (Model 6) and disc blackbody plus thermal Comptonisation of a blackbody (Model 7). The quality of the fits is similar for Models 4-6, confirming that the chosen models are degenerate in the available energy band. In fact, the degeneracy is such that Model 7 fails to converge due to a higher number of degrees of freedom compared to the other two-component models (and is therefore not shown).

Therefore, we next tried to further constrain the models by using hard X-ray information whenever available. For obs $2 \mathrm{~b}$ we used the simultaneous Swift and NuSTAR observations to constrain the fit (see below). For all other observations, we compared the X-ray light curves, hardness-intensity diagrams (HIDs) and X-ray spectra of the 2016 outburst and previous outbursts with broad-band X-ray observations. For the observations during the hard-to-soft transition, it is remarkable that the light curves and HIDs are almost identical for the 2016 and the 2011 outbursts (see Fig. 3), the latter of which was covered with Suzaku (Ono et al. 2017). Indeed, looking at the outburst classification discussed in Sect. 1, both belong to the same category of FRED and S-type or long-bright outbursts, although the 2016 outburst is brighter than the 2011 one in soft X-rays, constituting the most energetic outburst ever observed from Aql X-1 (Güngör et al. 2017).

It is apparent that obs 1 occurred at an epoch similar to the Suzaku observation in 2011 (obs 406010020, Ono et al. 2017) but ended a few hours before. Specifically, the Suzaku observation spanned from day 12.54 to day 13.46 (since the start of outburst, see Fig. 3), although the X-ray spectrum only evolved significantly after day 12.82 (interval P0, Ono et al. 2017). Our obs 1 spanned from day 12.44 to day 13.15 and obs 2 a started at a later epoch, on day 14.10. Thus, we expect that the spectra of obs 1 to $2 \mathrm{~b}$ resemble the spectra of the beginning/end of the Suzaku 2011 observation, marking the start and the end of the transition from a hard to a soft state. To verify that this is 
M. Díaz Trigo et al.: The evolving jet spectrum of Aql X-1
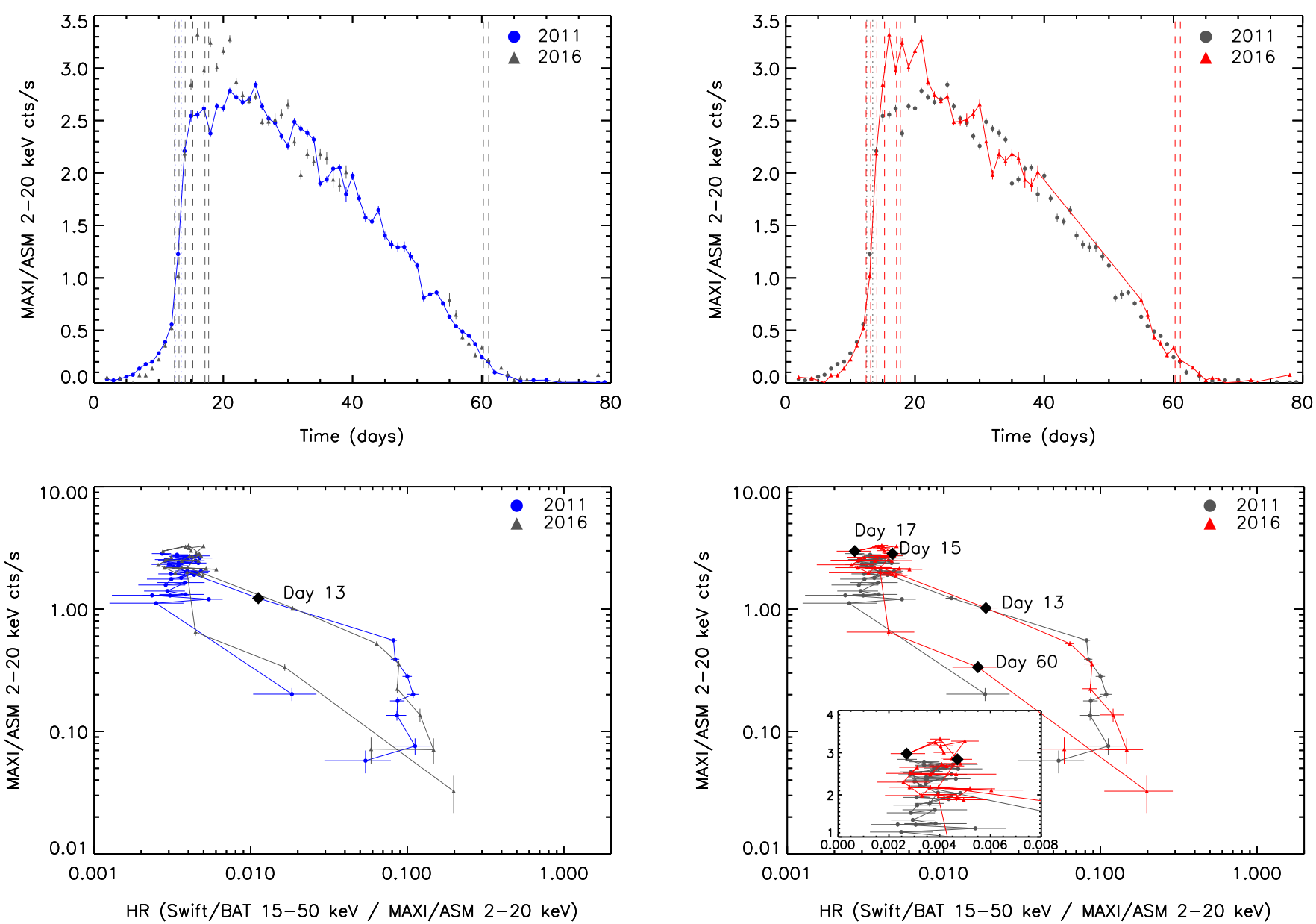

Fig. 3. MAXI 2-20 keV light curves (upper plots) and hardness-intensity diagrams (lower plots) for the 2011 and 2016 outbursts of Aql X-1. The light curves show days since the start of outburst (MJD 55843 and 57590 for the 2011 and 2016 outbursts, respectively). The vertical lines mark the time elapsed by the pointed observations: blue lines correspond to the Suzaku observations in 2011 and red to the observations in 2016 presented here. The black diamonds mark the day during which the pointed Suzaku observations during the 2011 outburst (lower-left) and the Swift/XRT observations listed in Table 1 (lower-right) were performed. The inset in the lower-right panel shows a zoom of the region of the HID where obs $2 \mathrm{a}$ and $2 \mathrm{~b}$ are placed. In the lower panels, only diamonds that have a hardness ratio with $>2 \sigma$ significance are shown.
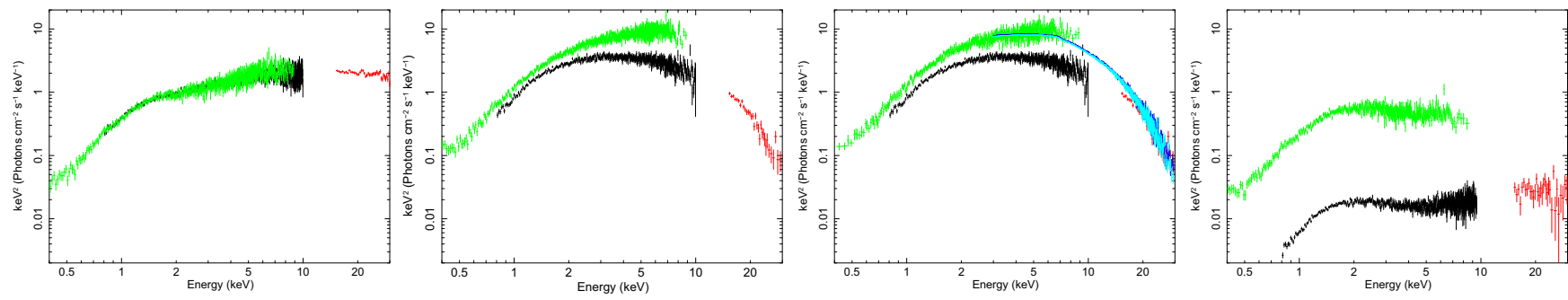

Fig. 4. Background-subtracted Swift/XRT spectra of AqlX-1 for obs 1 (left panel), 2a (middle-left panel), $2 \mathrm{~b}$ (middle-right panel) and 3 (right panel) after deconvolution with the response matrix using, as a model, a power law of index 2. In each panel, we show the $S w i f t / X R T$ spectra (in green) together with Suzaku broad-band spectra (in black and red) taken at similar epochs during outburst evolution. For obs $2 \mathrm{~b}$, we also show the NUSTAR simultaneous spectra (pink, light blue for FPMA/B, respectively). Based only on the comparison to broad-band spectra from previous outbursts, we identify obs 1 and 3 with hard states and obs $2 \mathrm{a}$ and $2 \mathrm{~b}$ with soft states (see text).

the case, we extracted the spectra from the Suzaku X-ray Imaging Spectrometer (XIS, Koyama et al. 2007) and the Hard X-ray Detector (PIN, Takahashi et al. 2007) instruments corresponding to periods P0 and P9 from Ono et al. (2017) following standard procedures. We compared them to the Swift and Swift/NuSTAR spectra of obs 1-2b by unfolding the spectra through the response matrix using a power law of index 2 (see Fig. 4). Our obs 1 perfectly matches the P0 interval from Ono et al. (2017), while our obs $2 \mathrm{a}$ and $2 \mathrm{~b}$ are at an even softer and brighter state than the P9 interval from Ono et al. (2017).

For obs 3, occurring during the soft-to-hard transition, we extracted the Suzaku spectra obtained during the decay of the 2007 outburst (Fig. 2, Sakurai et al. 2014). Obs 3 is still about twice the luminosity of the first Suzaku observations during the decay (obs 402053020, 402053030 and 402053040) but the spectral shape looks similar to an even later stage during the 
decay (obs 402053050, or Suzaku obs 5). Therefore, in Fig. 4 we plot obs 3 together with the latter observation from Sakurai et al. (2014).

Taking into account the similarities between Swift/XRT spectra and previous broad-band spectra highlighted above we next constrained the fits as follows: we used a basic model consisting of thermal Comptonisation absorbed from neutral material (tbabs*nthcomp in XSPEC) and, where necessary, we added a disc blackbody (tbabs*(diskbb+nthcomp)). For obs $2 b$, to account for the strong iron $\mathrm{K}$ line present in the NUSTAR spectra we also included the reflection component rfxconv (Kolehmainen et al. 2011) and the final model was tbabs*(diskbb+rfxconv*nthcomp). Then, we fitted obs 1 and Suzaku P0 tying all the parameters of the fit. Next, we fitted obs $2 \mathrm{~b}$ with the NUSTAR spectra again tying all the parameters of the fit. Finally, we fitted Suzaku P9 and Suzaku obs 5 individually. Since our obs $2 \mathrm{a}$ and 3 are not similar enough to or have simultaneous hard X-ray observations, we imposed values for the parameters intermediate between those of Suzaku P9 and obs $2 \mathrm{~b}$ and between obs $2 \mathrm{~b}$ and Suzaku obs 5, respectively. Table A. 2 shows the results of the fits.

\section{Results}

\subsection{Accretion state}

The aim of our multi-wavelength observations was to catch the source at state transitions, which is particularly challenging given that they typically only last between 1 and 4 days (see Table 1, Muñoz-Darias et al. 2014).

Based on the comparison of the Swift/XRT spectra with previous outbursts and on the fits in Sect. 2.4 we conclude that obs 1 is a hard state: it can be well matched to Suzaku P0, classified as a hard state, and it can be fitted with only a Comptonisation component with relatively hard photon index (see Table A.2). Instead, obs 2a is softer than Suzaku P9, classified as a soft state, and we need to include a second component in the fit consisting of a relatively hot disc $(2.11 \mathrm{keV})$ besides the Comptonisation component that has a softer photon index (2.00) and a lower electron temperature compared to obs 1 . Therefore, we conclude that obs $2 \mathrm{a}$ is a soft state. Obs $2 \mathrm{~b}$ is very similar to obs $2 \mathrm{a}$ and is placed in the same part of the HID diagram (see Fig. 3). Moreover, the broad-band fits, including the simultaneous NuSTAR data, show again a hot disc and a Comptonisation component with a soft photon index (2.31) and a low electron temperature. We therefore conclude that obs $2 b$ also occurs during a soft state. We also note that the Eddington fraction during obs $2 a / 2 b$ is $\sim 60-65 \%$ for a $1.4 M_{\odot}$ neutron star, compared to $30 \%$ at the end of the Suzaku observation in 2011. While the $2-20 \mathrm{keV}$ light curves seem to indicate that the peak of the outburst occurred between obs $2 \mathrm{a}$ and $2 \mathrm{~b}$, the $15-50 \mathrm{keV}$ flux already peaks during obs 1 and drops thereafter, causing a significant drop in the Swift/(BAT/XRT) HR by the time of obs 2a (see Fig. 1, panel d). In contrast, the OIR flux peaks a few days after obs 2 b (see Fig. 1, panel e).

The reverse transition, from a soft to a hard state, occurs generally when the source bolometric luminosity has decreased well below $10 \%$ Eddington and is marked by a sudden decrease of the soft X-rays, which is also associated with a change in the X-ray HR. In the 2016 outburst, we detected an increase in the 15-50 keV/0.3-10 keV HR around day 57, as the bolometric luminosity was $\sim 5 \%$. Obs 3 was then performed at days $60-61$ and it clearly lies in a relatively hard region of the HID. The fits from the previous section indicate that the temperature of the
Table 3. Accretion state and radio to millimetre spectral indices.

\begin{tabular}{llccc}
\hline \hline Obs. & State & Slope 1 & Slope 2 & Jet break \\
\hline 1 & Hard/Intermediate & $0.11 \pm 0.06$ & $-0.15 \pm 0.06$ & $\sim 100 \mathrm{GHz}$ \\
1 & Hard/Intermediate & $0.11 \pm 0.06$ & $-1.4 \pm 0.7$ & $\sim 250 \mathrm{GHz}$ \\
2a & Soft & $-1.76 \pm 0.25$ & $\lesssim 5 \mathrm{GHz}$ \\
2b & Soft & $-0.19 \pm 0.09$ & $\lesssim 5 \mathrm{GHz}$ \\
3 & Hard/Intermediate & $0.70 \pm 0.17$ & $-0.21 \pm 0.01$ & $\sim 30 \mathrm{GHz}$ \\
3 & Hard/Intermediate & $0.34 \pm 0.05$ & $-0.21 \pm 0.01$ & $\sim 100 \mathrm{GHz}$ \\
\hline
\end{tabular}

Notes. For obs 1 and 3, we obtained two jet spectral breaks per observation by taking into account different scenarios (see text). The two values are considered as lower and upper limits of the break for each observation. The true value very likely lies between these two extremes.

disc has significantly dropped to $0.57 \mathrm{keV}$ while the index of the Comptonisation component has become harder again (1.78). Thus, we classify obs 3 as a hard state.

Besides the spectral degeneracy, a caveat to the classification above is that the multi-wavelength observations span several hours. For example, obs $1 / 3$ last in total $17 / 19$ h, respectively, which corresponds to about twice the duration of the hard-to-soft transition observed by Ono et al. (2017). This is not a concern for obs $2 \mathrm{a} / 2 \mathrm{~b}$, which we can safely classify as soft states based on the high luminosity, the position in the HID and the curvature of the Swift/XRT-NuSTAR spectra. In contrast, obs 1/3 show Swift/XRT spectra consistent with a hard state (see above) based on a comparison with previous broad-band X-ray spectra, but the luminosity and the position in the HID may indicate that the transition is actually underway. The Swift/BAT to Swift/XRT HR has already started to decrease before the onset of obs 1 (see Fig. 1d). The Swift/BAT to MAXI HID shows that the daily HR is still on the hard region of the HID on day 12, at the time of the ATCA observation, but has significantly moved towards the soft region on day 13 (see Fig. 3, lower-right). Hence, ALMA observations, occurring on day $13,1.75 \mathrm{~h}$ before the Swift/XRT observation, could already track the start of the transition. Similarly, the Swift/BAT to Swift/XRT HR shows a peak during obs 3 (see Fig. 1d) but decreases right after (although the errors are large). The Swift/BAT to MAXI HID shows that the daily $\mathrm{HR}$ is moving towards the hard region of the HID on day 60 and has reached this region by the next day (see Fig. 3, lowerright). Specifically, the ATCA observation could track a state softer than the Swift/XRT observation, starting $3.2 \mathrm{~h}$ later during day 60 . The ALMA observations, starting $7.7 \mathrm{~h}$ after the end of the Swift/XRT observation, should track a slightly harder state than the latter observation.

Therefore, we conclude that obs $1 / 3$ may be tracking a relatively hard state, but during the state transition, and classify them as hard/intermediate states. Table 3 summarises the state classification of our multi-wavelength pointed observations taking into account these considerations.

\subsection{Broad-band spectra}

We constructed broad-band spectra for the four pointed observations (see Fig. 5). The radio to millimetre spectra for obs 1 and 3 are relatively similar in shape, with the spectral slope being positive from radio to millimetre frequencies, as expected from optically thick synchrotron emission from a jet. A spectral break occurs at frequencies of $\sim 100 \mathrm{GHz}$ and the spectral slope becomes negative above that frequency. The mid-IR upper limit 

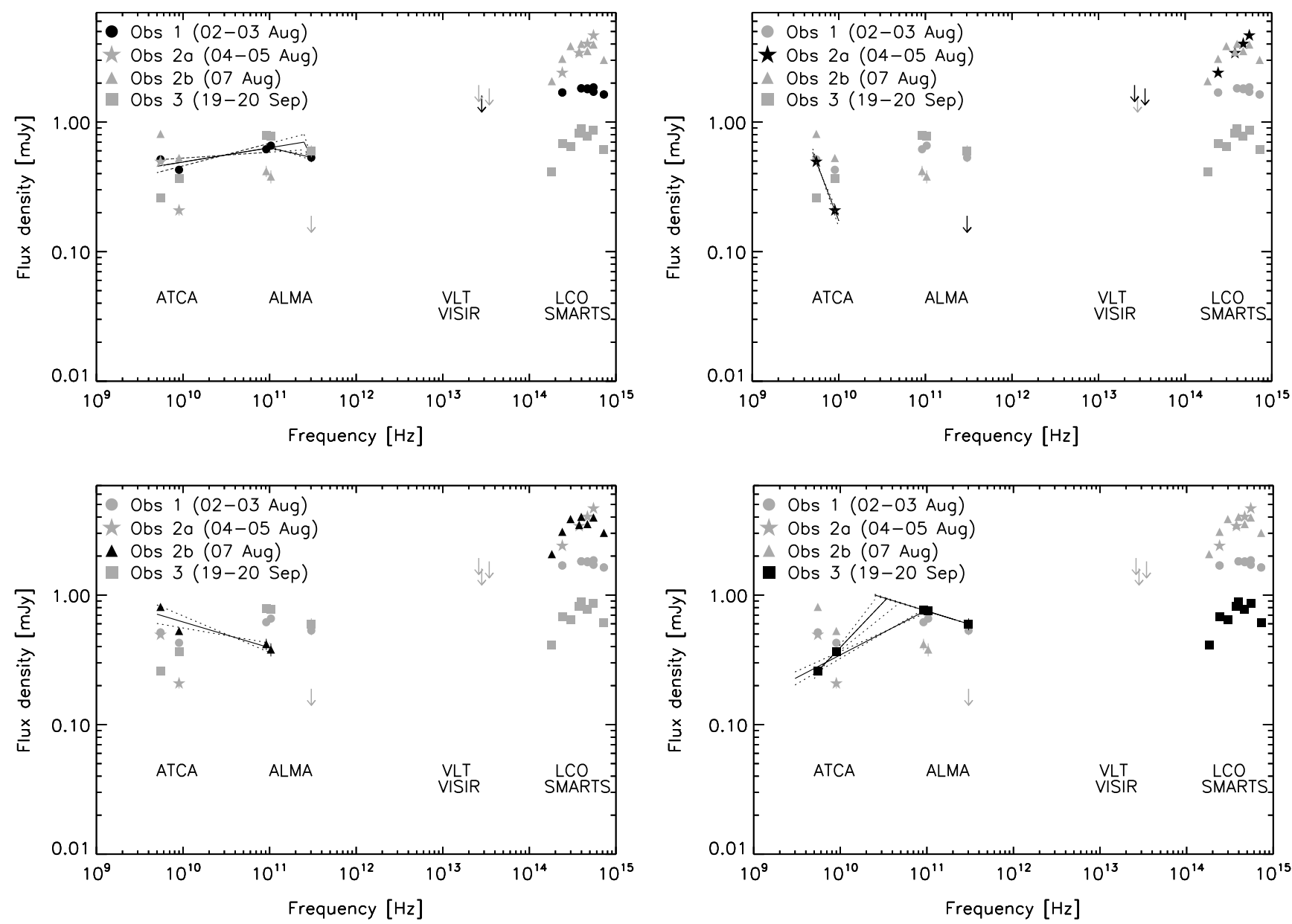

Fig. 5. Broad-band spectra for Aq1 X-1 for obs 1-3. The arrows represent $3 \sigma$ upper limits. Obs 1, 2a, $2 \mathrm{~b}$ and 3 are shown in black in the upper-left, upper-right, lower-left and lower-right panels, respectively. For obs 3 we show two possible fits for the first slope: considering only the ATCA points and considering the ATCA points together with the ALMA points. The jet frequency break could be anywhere between $\sim 30 \mathrm{GHz}$ and $100 \mathrm{GHz}$ depending on the solution taken. The jet break frequency evolves from $\sim 100 \mathrm{GHz}$ in obs 1 , to $\lesssim 5 \mathrm{GHz}$ in obs $2 \mathrm{a}$ and $2 \mathrm{~b}$ and back to $30-100 \mathrm{GHz}$ in obs 3 .

obtained for obs 1 is consistent with this scenario. In contrast, the spectra for obs $2 \mathrm{a}$ and $2 \mathrm{~b}$ show a negative spectral slope from radio to millimetre frequencies, that is, the peak of emission occurs at the lowest observed frequency, 5.5 GHz. Interestingly, obs $2 \mathrm{~b}$ shows stronger flux densities compared to obs $2 \mathrm{a}$, possibly indicating a jet re-brightening. The spectral slope is steeper below $9 \mathrm{GHz}$ than between 9 and $97.49 \mathrm{GHz}$ for obs $2 \mathrm{~b}$. Obs $2 \mathrm{a}$ could in principle also show two different spectral slopes below and above $9 \mathrm{GHz}$ but we cannot determine if this is really the case due to the flux upper limit at $302.99 \mathrm{GHz}$. The OIR spectra show a significant increase in flux from obs 1 to obs $2 a / 2 b$ and the smallest flux is present in obs 3 .

We next fitted the spectral slopes for all the radio to millimetre spectra. For obs 1 and 3, a break at $\sim 100 \mathrm{GHz}$ is apparent (see Fig. 5). Therefore, we used the first four points/last three points to determine the spectral slope before/after the break. For obs 1 , we obtain indices of $0.11 \pm 0.06$ and $-0.15 \pm 0.06$ before and after the break, respectively, where $F_{v} \propto v^{\alpha}$ and $F_{v}$ is the flux density at frequency $v$. These indices are consistent with the spectrum being the result of optically thick/optically thin synchrotron emission from a jet, before and after the break, respectively. Similarly, for obs 3, we obtain indices of $0.34 \pm 0.05$ and $-0.21 \pm 0.01$ before and after the break at $\sim 100 \mathrm{GHz}$, respectively. For this observation, the break could lie instead at frequencies as low as $\sim 30 \mathrm{GHz}$, if we consider the optically thick slope traced by the ATCA points only. In contrast, for obs 1 , the ATCA points trace a negative slope, which could indicate some structure in the broad-band spectra, unaccounted-for systematic uncertainties, or time variability. Also, the break could lie at frequencies higher than $100 \mathrm{GHz}$ if we assume a steeper slope after the break. Specifically, to set a tentative upper limit on the frequency break for obs 1 , we calculated the slope of the optically thin spectrum assuming an extension of the optically thick spectrum to 200 and $250 \mathrm{GHz}$. We obtained values of $-0.6 \pm 0.3$ and $-1.4 \pm 0.7$, respectively, for that slope. Therefore, we set a conservative upper limit of $250 \mathrm{GHz}$ for the break frequency but note that this would already yield a very extreme slope, similar to that of obs $2 \mathrm{a}$ (see below), and is therefore unlikely. For obs $2 \mathrm{a}$ we obtain a spectral slope of $-1.76 \pm 0.25$ considering only the 5.5 and $9 \mathrm{GHz}$ detections. Considering the ATCA detections and the ALMA $3 \sigma$ upper limit we obtain an upper limit on the spectral slope of -0.19 . For obs $2 b$ we obtain a spectral slope of $-0.20 \pm 0.08$ between 5 and $97.49 \mathrm{GHz}$. Interestingly, for this observation the slope traced by the ATCA points only is significantly steeper than when fitting a single power law to both the ATCA and the ALMA points. Similarly to obs 1, this could indicate again some structure in the broad-band spectra. However, the different slopes at radio and millimetre frequencies could be simply explained by variability between the observations that are not strictly simultaneous. 


\section{Discussion}

\subsection{Jet emission during soft states}

The evolution of the radio emission across outbursts was first systematically studied for Aql X-1 by Tudose et al. (2009) for the outbursts from 2002, 2004, and 2005. They reported a significant drop in radio fluxes above a bolometric flux of $5 \times$ $10^{-9} \mathrm{erg} \mathrm{cm}^{-2} \mathrm{~s}^{-1}$ (corresponding to $10 \%$ of the Eddington luminosity at a distance of $5.2 \mathrm{kpc}$ ) and suggested that such a drop could be analogous to the suppression of radio emission in $\mathrm{BH}$ XRBs in soft, bright, X-ray states. Miller-Jones et al. (2010) also observed a quenching of radio emission ( $3 \sigma$ upper limits of 0.17 and $0.08 \mathrm{mJy}^{\text {beam }}{ }^{-1}$ at 4.8 and $8.4 \mathrm{GHz}$, respectively) at bolometric fluxes larger than $10 \%$ of Eddington during the November 2009 outburst (classified as S-type, Asai et al. 2012 or short-low, Güngör et al. 2014, respectively).

In contrast, we detect radio emission during obs $2 \mathrm{a}$ and both radio and millimetre emission during obs $2 \mathrm{~b}$, at bolometric fluxes of 60 and $65 \%$ Eddington, respectively, and with X-ray spectra consistent with a soft state. It is also remarkable that although the radio flux has dropped from obs 1 to $2 \mathrm{a}$, during obs $2 \mathrm{~b}$ we find a flux density of $816 \pm 35 \mu \mathrm{Jy}$ at $5.5 \mathrm{GHz}$, the highest ever observed at this frequency. Moreover, the spectra of obs $2 \mathrm{a}$ and $2 \mathrm{~b}$ show a negative slope. Based on the monitoring of the 2009 outburst, Miller-Jones et al. (2010) showed that the spectral slope at radio frequencies was consistent with being flat outside of the hard state, that is, consistent with optically thick emission, and suggested that the internal shock mechanism believed to produce optically thin transient radio ejecta was not active in AqlX-1. While a negative slope of $-0.80 \pm 0.35$ was detected between 5.25 and $7.45 \mathrm{GHz}$ with the VLA during the 2013 outburst (of long-high type, Güngör et al. 2014) at the hard-to-soft transition, the large flux uncertainty did not allow to confirm the presence of optically thin flares (Miller-Jones \& Sivakoff 2013). Our observations $2 \mathrm{a}$ and $2 \mathrm{~b}$ demonstrate the existence of radio to millimetre emission with a negative slope after the source has transited into the soft state and at luminosity levels of $60-65 \%$ Eddington, well above the previous limits.

Radio emission with negative slopes from $\mathrm{BH}$ transients during soft states has been detected in a handful of sources (Corbel et al. 2004; Neilsen et al. 2014) and interpreted in a scenario where the core jet is strongly suppressed and the emission is associated with optically thin emission from re-brightenings at shocks as the jet propagates away from the binary (Fender et al. 2009). In such a scenario the emission should monotonically fade as the ejecta expand in the surrounding medium, unless new ejecta are launched. While the existence of a core jet during the soft state cannot be ruled out, it would then be difficult to explain why the observed emission is optically thin, as opposed to that of the hard state. Indeed, the spectral shape of the jet does not significantly change (only the intensity) in models invoking the existence of a core jet during soft states (Malzac 2014; Drappeau et al. 2017). Our observations show that, similarly to $\mathrm{BHs}$, NSs can also show radio to millimetre emission shortly after the transition to the soft state with a negative slope. Interestingly, obs 2 a shows a very extreme spectral index, albeit based on only two ATCA points. Such a steep slope, with an index $<-1.5$, has only seldom been observed previously (Fender et al. 2009; van der Horst et al. 2013) and at least in one case it has been associated to a spectral break at radio frequencies. While such indices seem too steep to be attributed to standard optically thin synchrotron, it might be that the emission evolves on different timescales at different frequencies or that the electron distribution has a sharp cutoff.
Obs $2 \mathrm{~b}$ shows a less negative spectral index than obs $2 \mathrm{a}$. However, there seems to be some structure in the spectrum, namely the slope derived from the ATCA points only is much steeper (with an index of -0.87) than when the ATCA and ALMA points are considered. This could be due to variability between the ALMA and ATCA observations, taken $8 \mathrm{~h}$ apart.

Regardless of the spectral slope, it is clear that it is negative, similar to BHs and in contrast to the optically thick emission detected from persistent NSs in a steady soft state (Migliari et al. 2004; Díaz Trigo et al. 2017). This implies that two different types of jet emission may be present during soft states of NSs: optically thin emission in transient sources and optically thick emission in steady states of persistent sources. We note that in some $\mathrm{BH}$ sources, several radio flares from discrete ejecta have been seen to be launched within days of the hard to soft transition (e.g. XTE J1859+226; Swift J1745-26; Brocksopp et al. 2002; Fender et al. 2009; Curran et al. 2014). It is possible that because of the rapid transition of Aql X-1, and the sparse radio coverage, some radio flares may have been missed. Another possibility is that the flares are associated only to the brightest outbursts. While the 2011, 2013, and 2016 outbursts all belong to the "long-high" class based on the duration and maximum flux of the X-ray emission (Güngör et al. 2014), the 2002 and the 2009 outbursts belong to a different, less bright category.

\subsection{Evolution of the jet spectral break along the outburst}

During the last few years, major effort has been devoted to determining what drives the jet ejection and reignition during spectral state transitions, whether jet ejections are always launched, and whether a weak compact jet might be present at all during soft states. As discussed above, the emission observed during soft states has been generally attributed to optically thin emission from jet ejecta. However, there is at least one BH, MAXI J1659-152, for which radio emission with a very steep negative slope has been detected in a soft state (van der Horst et al. 2013) despite the absence of detections of ejecta with e-VLBI (Paragi et al. 2013). Moreover, similar optically thin emission has also been detected for the BH GX 339-4 at the reverse transition from soft to hard states before the appearance of an optically thick jet (Corbel et al. 2013). In both cases, the break frequency is below $\sim 10 \mathrm{GHz}$ and seems to indicate that the negative slope spectra may also provide clues as to the mechanism by which jets switch on and off. Perhaps the clearest indication of this is the continuous evolution of the spectral break towards lower frequencies and back to higher frequencies in the $\mathrm{BH}$ MAXI J1836-194 as the outburst evolves from a hard to an intermediate state and back (Russell et al. 2014). At least in this case, the evolution is continuous and even if the transition to a soft state never occurs, optically thin emission is observed down to a break frequency of $\lesssim 30 \mathrm{GHz}$. Moreover, the break frequency is directly correlated to the hardness of the X-ray spectrum. Interestingly, such a correlation is also found when the comparison is extended to super-massive BHs (Koljonen et al. 2015).

The few break frequencies detected to date in NSs in hard states lie in the IR band (Migliari et al. 2010; Baglio et al. 2016; Díaz Trigo et al. 2017). Our observations show for the first time the temporal evolution of the break frequency for a NS. The frequency of the break in obs 1 is at a lower frequency than that of previously detected breaks during hard states. While we cannot discard that the break lies at this low frequency in hard states well before the transition, it seems plausible that the low frequency is a result of the evolution at the start of the spectral 

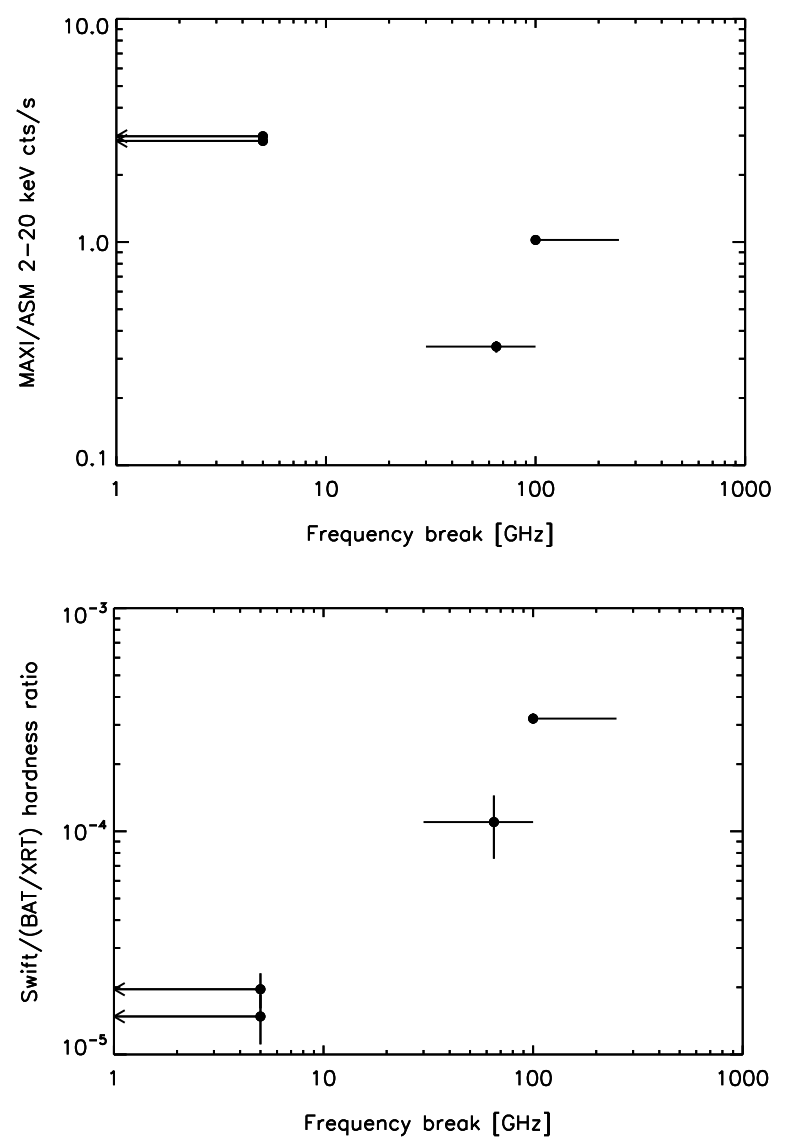

Fig. 6. Frequency of the jet spectral break versus the MAXI/ASM Xray count rate (top panel) and the Swift/BAT/XRT HR (bottom panel) for obs $1-3$.

transition. This observation highlights the importance of truly broad-band coverage for jet studies since based on the radio spectra alone we would have concluded that the spectral break was already below $5 \mathrm{GHz}$ at this stage. Since the ALMA observations were performed after the ATCA ones, we can discard that the break moved towards lower frequencies between the ATCA and the ALMA observations.

Following the transition to a soft state, both obs $2 \mathrm{a}$ and $2 \mathrm{~b}$ show a steep spectrum with no apparent break in the frequency range observed. Therefore, we conclude that if the compact jet still exists, the break must be below $\sim 5 \mathrm{GHz}$. The re-brightening during obs $2 b$ could be attributed to an enhanced episode of mass channelled into the jet. For this to happen, either a core jet should still be present during obs $2 \mathrm{a}$ or the core jet should switch off and on between obs $2 \mathrm{a}$ and $2 \mathrm{~b}$. Alternatively, the re-brightening could be due to a new discrete ejection, a shock between ejected material and the ISM, or even the interaction of two ejecta at different speeds.

During obs 3 , the jet break is detected at $\sim 30-50 \mathrm{GHz}$ (if we consider only the ATCA points) or $\sim 100 \mathrm{GHz}$ (if we fit the ATCA and ALMA low-frequency points together; see Fig. 5). Should the break frequency be lower at the same hardness at the outburst decay, this could be due to the reduced mass accretion rate (Heinz \& Sunyaev 2003; Falcke et al. 2004). However, we cannot determine if this is the case from these observations. Interestingly, the flux densities detected at millimetre frequencies are the highest from all observations indicating that the millimetre emission is not directly correlated with the soft or hard X-ray flux and that a powerful jet can be present during the decay of the outburst. After obs 3, we would expect that the jet evolves by reigniting at higher frequencies up to OIR (e.g. Corbel et al. 2013). However, for Aql X-1, the contribution of the jet at OIR frequencies is probably not dominant (if existent at all) during hard states, as opposed, for example, to the persistent neutron star 4U 1728-34 (Díaz Trigo et al. 2017), since we do not see any significant re-brightening at such frequencies during the decay. Alternatively, the jet could have decayed too quickly to be detectable at any frequency after obs 3.

Finally, we explored if the break frequency could be directly correlated to the hardness of the X-ray spectrum, as has recently been found for BHs (Russell et al. 2014; Koljonen et al. 2015). Figure 6 shows the break frequencies obtained for the four multi-wavelength observations versus the MAXI/ASM 2-20 keV count rate and the Swift/BAT/XRT HR. For obs $1 / 2 \mathrm{a}$, we use the flux/HR during days $13 / 15$, respectively, since these are the days when the Swift/XRT and ALMA observations were performed and note that at least the ATCA observation on day 12 may have happened during a slightly harder state than the corresponding Swift/XRT and ALMA ones. For obs 3, we use the flux/HR during day 60 since all observations were performed on that day except the ALMA band 7 one, which was performed very close, on day 61.04. Similarly to what was observed for BHs, the frequency of the break increases with hardness of the X-ray spectra. While a Spearman rank test yields a positive correlation of $90 \%$ between the jet break frequency and the hardness of the $\mathrm{X}$-ray spectrum, such a correlation still has a chance probability $>5 \%$ given the low number of degrees of freedom (2). Therefore, more data are needed to confirm this correlation in Aql X-1. A correlation of the break frequency with the X-ray flux is not observed. A caveat regarding this comparison is that, in NSs, the X-ray spectrum shows a significant contribution of the NS surface or boundary layer. Therefore, an interpretation of the dependence of the frequency break as a function of spectral components such as the accretion disc or hot corona is more difficult than for BHs.

\section{Conclusions}

We observed the NS Aql X-1 across the hard-to-soft transition and during the decay of its 2016 outburst from radio to X-rays. We detected radio emission in all cases, which comprise both hard/intermediate and soft accretion states. Millimetre emission was also detected in three out of four observations, including one soft state. The broad-band spectra from radio to millimetre frequencies evolve from having a spectral break from optically thick to thin emission at $\sim 100 \mathrm{GHz}$ during hard/intermediate states to below $\sim 5 \mathrm{GHz}$ during soft states assuming the compact jet persists. We do not find any correlation of the X-ray flux with the spectral break. In contrast, a correlation of the break frequency with X-ray spectral hardness is possible, like that discovered in $\mathrm{BH}$ transients. These observations show that the processes at play in $\mathrm{BH}$ jets are also present in NS transients and highlight the importance of simultaneous multi-wavelength coverage of XRB outbursts.

Acknowledgements. This paper makes use of the following ALMA data: ADS/JAO.ALMA\#2015.1.00734.T. ALMA is a partnership of ESO (representing its member states), NSF (USA) and NINS (Japan), together with NRC (Canada) and NSC and ASIAA (Taiwan), in cooperation with the Republic of Chile. The Joint ALMA Observatory is operated by ESO, AUI/NRAO and NAOJ. The Australia Telescope Compact Array is part of the Australia Telescope National Facility which is funded by the Australian Government for operation as a National Facility managed by CSIRO. D. A. acknowledges support from the Royal Society. J. C. A. M.-J. is the recipient of an Australian Research Council Future Fellowship (FT140101082). D. M. R. thanks Mario van den 
Ancker at ESO for help with the preparation and flux calibration of the VLT/VISIR observations. The Faulkes Telescope Project is an education partner of Las Cumbres Observatory. The Faulkes Telescopes are maintained and operated by LCO.

\section{References}

Arnaud, K. A. 1996, in Astronomical Data Analysis Software and Systems V, ASP Conf. Ser., 101, 17

Asai, K., Matsuoka, M., Mihara, T., et al. 2012, PASJ, 64, 128

Baglio, M. C., D’Avanzo, P., Campana, S., et al. 2016, A\&A, 587, A102

Brocksopp, C., Fender, R. P., McCollough, M., et al. 2002, MNRAS, 331, 765

Buxton, M. M., Bailyn, C. D., Capelo, H. L., et al. 2012, AJ, 143, 130

Campana, S., Brivio, F., Degenaar, N., et al. 2014, MNRAS, 441, 1984

Cardelli, J. A., Clayton, G. C., \& Mathis, J. S. 1989, ApJ, 345, 245

Casella, P., Altamirano, D., Patruno, A., Wijnands, R., \& van der Klis, M. 2008, ApJ, 674, L41

Chevalier, C., \& Ilovaisky, S. A. 1991, A\&A, 251, L11

Chevalier, C., Ilovaisky, S. A., Leisy, P., \& Patat, F. 1999, A\&A, 347, L51

Condon, J. J., Cotton, W. D., Greisen, E. W., et al. 1998, AJ, 115, 1693

Corbel, S., Fender, R. P., Tomsick, J. A., Tzioumis, A. K., \& Tingay, S. 2004, ApJ, 617, 1272

Corbel, S., Aussel, H., Broderick, J. W., et al. 2013, MNRAS, 431, L107

Coriat, M., Corbel, S., Prat, L., et al. 2011, MNRAS, 414, 677

Curran, P. A., Coriat, M., Miller-Jones, J. C. A., et al. 2014, MNRAS, 437, 3265

DePoy, D. L., Atwood, B., Belville, S. R., et al. 2003, in Instrument Design and Performance for Optical/Infrared Ground-based Telescopes, eds. M. Iye \& A. F. M. Moorwood, Proc. SPIE., 4841, 827

Díaz Trigo, M., Migliari, S., Miller-Jones, J. C. A., et al. 2017, A\&A, 600, A8

Drappeau, S., Malzac, J., Coriat, M., et al. 2017, MNRAS, 466, 4272

Evans, P. A., Beardmore, A. P., Page, K. L., et al. 2007, A\&A, 469, 379

Falcke, H., Körding, E., \& Markoff, S. 2004, A\&A, 414, 895

Fender, R. P., Belloni, T. M., \& Gallo, E. 2004, MNRAS, 355, 1105

Fender, R. P., Homan, J., \& Belloni, T. M. 2009, MNRAS, 396, 1370

Foight, D. R., Güver, T., Özel, F., \& Slane, P. O. 2016, ApJ, 826, 66

Galloway, D. K., Ajamyan, A. N., Upjohn, J., \& Stuart, M. 2016, MNRAS, 461, 3847

Gandhi, P., Blain, A. W., Russell, D. M., et al. 2011, ApJ, 740, L13

Gehrels, N., Chincarini, G., Giommi, P., et al. 2004, ApJ, 611, 1005

Güngör, C., Güver, T., \& Ekşi, K. Y. 2014, MNRAS, 439, 2717

Güngör, C., Ekşi, K. Y., \& Göğüş, E. 2017, New Ast., 56, 1

Heinz, S., \& Sunyaev, R. A. 2003, MNRAS, 343, L59

Henden, A. A., Levine, S., Terrell, D., \& Welch, D. L. 2015, Am. Astron. Soc. Meet. Abstracts, 225, 336.16

Hjellming, R. M., Han, X., \& Roussel-Dupre, D. 1990, IAU Circ., 5112

Jonker, P. G., \& Nelemans, G. 2004, MNRAS, 354, 355

Kalemci, E., Tomsick, J. A., Buxton, M. M., et al. 2005, ApJ, 622, 508

Kalemci, E., Dinçer, T., Tomsick, J. A., et al. 2013, ApJ, 779, 95

King, A. L., Tomsick, J. A., Miller, J. M., et al. 2016, ApJ, 819, L29

Kolehmainen, M., Done, C., \& Díaz Trigo, M. 2011, MNRAS, 416, 311

Koljonen, K. I. I., Russell, D. M., Fernández-Ontiveros, J. A., et al. 2015, ApJ, 814,139

Koyama, K., Inoue, H., Makishima, K., et al. 1981, ApJ, 247, L27

Koyama, K., Tsunemi, H., Dotani, T., et al. 2007, PASJ, 59, 23

Krimm, H. A., Holland, S. T., Corbet, R. H. D., et al. 2013, ApJS, 209, 14

Lagage, P. O., Pel, J. W., Authier, M., et al. 2004, The Messenger, 117, 12

Landolt, A. U. 1992, AJ, 104, 340
Lewis, F., Roche, P., Russell, D. M., \& Fender, R. P. 2008, in A Population Explosion: The Nature \& Evolution of X-ray Binaries in Diverse Environments, eds. R. M. Bandyopadhyay, S. Wachter, D. Gelino, \& C. R. Gelino, AIP Conf. Proc., 1010, 204

Lin, D., Remillard, R. A., \& Homan, J. 2007, ApJ, 667, 1073

Magnier, E. A., Schlafly, E., Finkbeiner, D., et al. 2013, ApJS, 205, 20

Maitra, D., \& Bailyn, C. D. 2008, ApJ, 688, 537

Malzac, J. 2014, MNRAS, 443, 299

Martí-Vidal, I., Vlemmings, W. H. T., Muller, S., \& Casey, S. 2014, A\&A, 563 , A136

Mata Sánchez, D., Muñoz-Darias, T., Casares, J., \& Jiménez-Ibarra, F. 2017, MNRAS, 464, L41

McMullin, J. P., Waters, B., Schiebel, D., Young, W., \& Golap, K. 2008, in Astronomical Data Analysis Software and Systems XVI, eds. R. A. Shaw, F. Hill, \& D. J. Bell, ASP Conf. Ser., 376, 127

Migliari, S., \& Fender, R. P. 2006, MNRAS, 366, 79

Migliari, S., Fender, R. P., Rupen, M., et al. 2004, MNRAS, 351, 186

Migliari, S., Tomsick, J. A., Miller-Jones, J. C. A., et al. 2010, ApJ, 710, 117

Miller-Jones, J. C. A., \& Sivakoff, G. R. 2013, ATel, 5148

Miller-Jones, J. C. A., Sivakoff, G. R., Altamirano, D., et al. 2010, ApJ, 716, L109

Mirabel, I. F., \& Rodriguez, L. F. 1994, Nature, 371, 46

Mitsuda, K., Inoue, H., Koyama, K., et al. 1984, PASJ, 36, 741

Muñoz-Darias, T., Fender, R. P., Motta, S. E., \& Belloni, T. M. 2014, MNRAS, 443, 3270

Neilsen, J., Coriat, M., Fender, R., et al. 2014, ApJ, 784, L5

Ono, K., Makishima, K., Sakurai, S., et al. 2017, PASJ, 69, 23

Paragi, Z., van der Horst, A. J., Belloni, T., et al. 2013, MNRAS, 432, 1319

Reig, P., van Straaten, S., \& van der Klis, M. 2004, ApJ, 602, 918

Romano, P., Campana, S., Chincarini, G., et al. 2006, A\&A, 456, 917

Russell, D. M., \& Lewis, F. 2009, ATel, 1970

Russell, D. M., Roche, P., Lewis, F., \& Maitra, D. 2010, ATel, 2871

Russell, D. M., Miller-Jones, J. C. A., Maccarone, T. J., et al. 2011, ApJ, 739, L19

Russell, D. M., Russell, T. D., Miller-Jones, J. C. A., et al. 2013, ApJ, 768, L35

Russell, T. D., Soria, R., Miller-Jones, J. C. A., et al. 2014, MNRAS, 439, 1390

Sakurai, S., Yamada, S., Torii, S., et al. 2012, PASJ, 64, 72

Sakurai, S., Torii, S., Noda, H., et al. 2014, PASJ, 66, 10

Sari, R., Piran, T., \& Narayan, R. 1998, ApJ, 497, L17

Sault, R. J., Teuben, P. J., \& Wright, M. C. H. 1995, in Astronomical Data Analysis Software and Systems IV, eds. R. A. Shaw, H. E. Payne, \& J. J. E. Hayes, ASP Conf. Ser., 77, 433

Skrutskie, M. F., Cutri, R. M., Stiening, R., et al. 2006, AJ, 131, 1163

Subasavage, J. P., Bailyn, C. D., Smith, R. C., et al. 2010, Proc. SPIE, 7737, $77371 \mathrm{C}$

Takahashi, T., Abe, K., Endo, M., et al. 2007, PASJ, 59, 35

Tingay, S. J., Jauncey, D. L., Preston, R. A., et al. 1995, Nature, 374, 141

Tody, D. 1986, in Instrumentation in Astronomy VI, ed. D. L. Crawford, Proc. SPIE, 627, 733

Tody, D. 1993, in Astronomical Data Analysis Software and Systems II, eds. R. J. Hanisch, R. J. V. Brissenden, \& J. Barnes, ASP Conf. Ser., 52, 173

Tudose, V., Fender, R. P., Linares, M., Maitra, D., \& van der Klis, M. 2009, MNRAS, 400, 2111

van der Horst, A. J., Curran, P. A., Miller-Jones, J. C. A., et al. 2013, MNRAS, 436, 2625

Verner, D. A., Ferland, G. J., Korista, K. T., \& Yakovlev, D. G. 1996, ApJ, 465, 487

Welsh, W. F., Robinson, E. L., \& Young, P. 2000, AJ, 120, 943

Wilms, J., Allen, A., \& McCray, R. 2000, ApJ, 542, 914

Zdziarski, A. A., Johnson, W. N., \& Magdziarz, P. 1996, MNRAS, 283, 193

Życki, P. T., Done, C., \& Smith, D. A. 1999, MNRAS, 309, 561 


\section{Appendix A}

Table A.1. Best fits to the 0.4-9 keV Swift/XRT spectra for observations 1-3 using one- and two-component models.

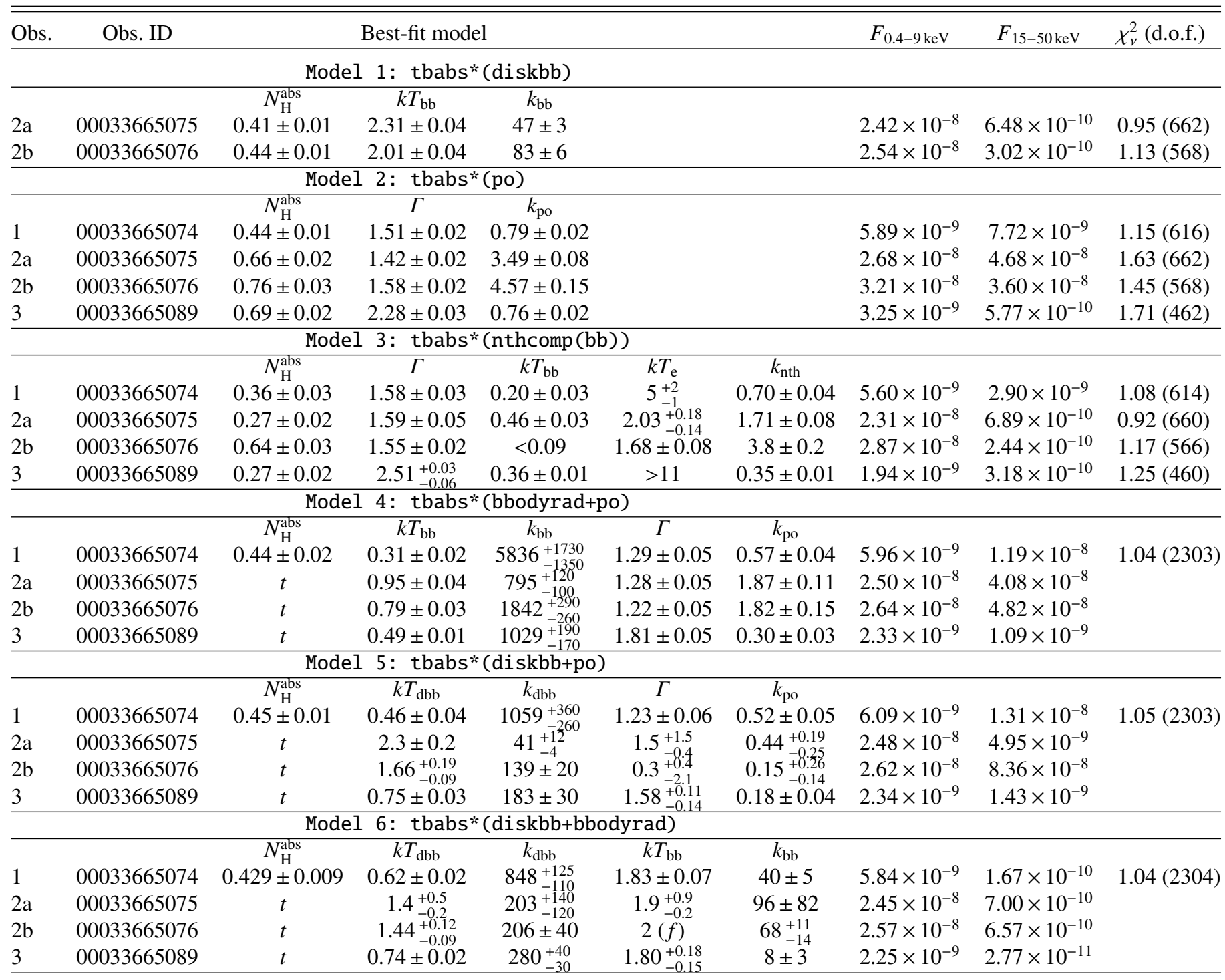

Notes. Only fits with $\chi_{v}^{2}<2$ are shown. $N_{\mathrm{H}}^{\mathrm{abs}}$ is the column density for the neutral absorber in units of $10^{22} \mathrm{~cm}^{-2} . k_{\mathrm{bb}}, k T_{\mathrm{dbb}}$ and $k_{\mathrm{po}}$ are the normalisations of the blackbody, disc blackbody and the power-law components in units of $\left[\left(R_{\mathrm{in}} / D_{10}\right)^{2}\right],\left[\left(R_{\mathrm{in}} / D_{10}\right)^{2} \cos \theta\right]$ and $\left[\mathrm{ph} \mathrm{keV}^{-1} \mathrm{~cm}^{-2} \mathrm{~s}^{-1}\right.$ at $1 \mathrm{keV}$ ], respectively, $R_{\mathrm{in}}$ being the radius in $\mathrm{km}, D_{10}$ the distance in units of $10 \mathrm{kpc}$ and $\theta$ the inclination of the source. $k_{\mathrm{nth}}$ is the normalisation of the nthcomp component. $k T_{\mathrm{bb}} / k T_{\mathrm{dbb}} / k T_{\mathrm{e}}$ are the temperatures of the blackbody/disc blackbody components and electrons in units of keV and $\Gamma$ the index of the power law. $F$ is the unabsorbed flux of the continuum in units of $\mathrm{erg} \mathrm{cm}^{-2} \mathrm{~s}^{-1} . t$ indicates that a parameter was tied for all the observations. $f$ indicates that a parameter was fixed during the fits. 
Table A.2. Best fits to the 0.4-9 keV Swift/XRT spectra for observations 1-3 using model tbabs*nthcomp for obs 1 and 3, model tbabs*(diskbb+nthcomp) for obs $2 \mathrm{a}$ and model tbabs*(diskbb+rfxconv*nthcomp) for obs $2 \mathrm{~b}$.

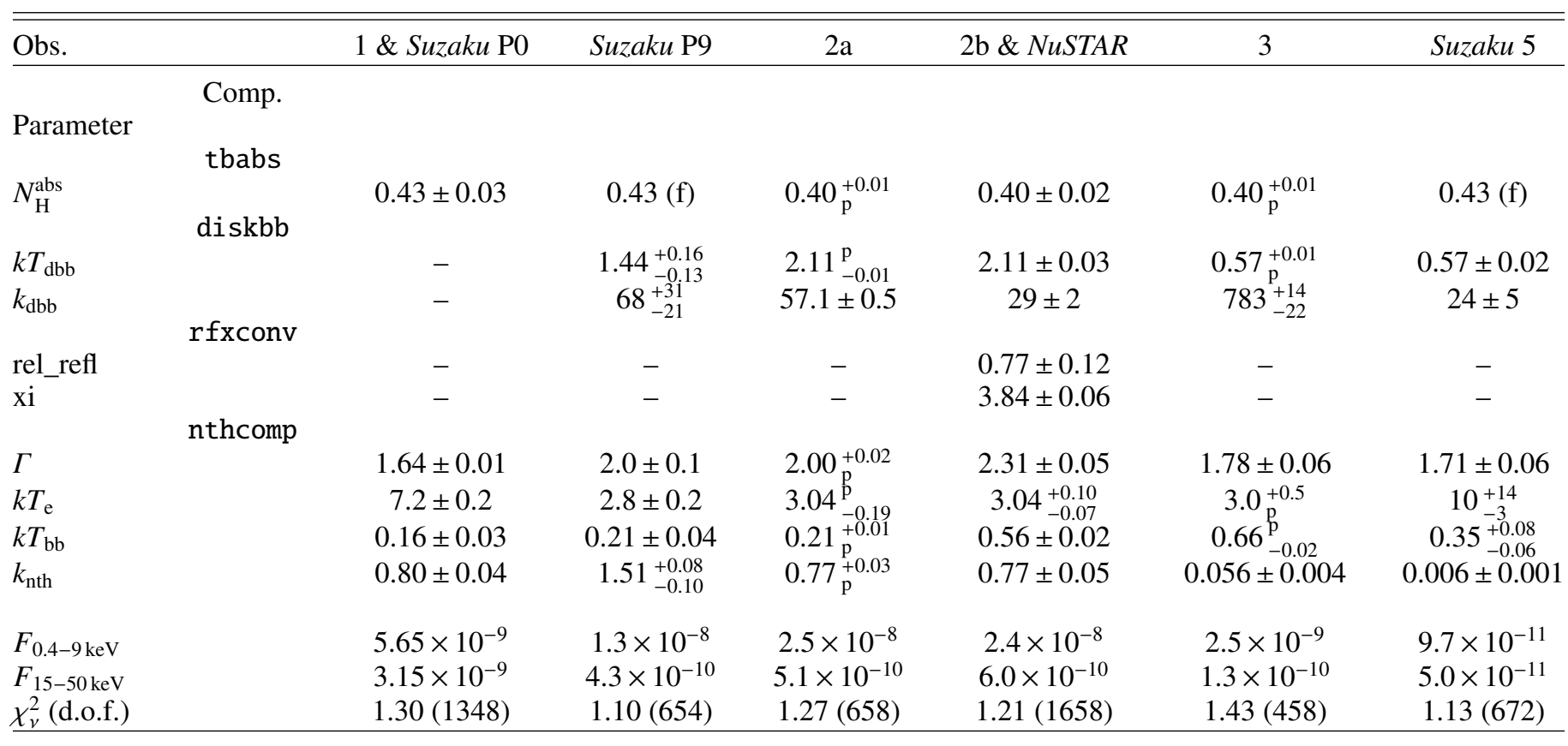

Notes. The model parameters are the same as in Table A.1. In addition, rel_refl is the reflection fraction and $\log (\xi)$ the ionisation parameter of the reflection component, for which we have fixed the inclination to $40^{\circ} . p$ indicates that a parameter pegged at its lower or upper limit. $f$ indicates that a parameter was fixed during the fits. For obs $2 \mathrm{a}$ and 3 we imposed values for the parameters of the fit between those of adjacent broad-band observations, except for the normalisations of the individual components in obs 3 that we leave free (see text). 